\title{
XIII Canadian Congress of Neurological Sciences XIII Congres Canadien Des Sciences Neurologiques
}

\author{
Vancouver, British Columbia \\ June $21-24,1978$
}

\author{
PARTICIPATING SOCIETIES/SOCIETES PARTICIPANTES \\ Canadian Neurosurgical Society \\ Société canadienne de neurochirurgie \\ Canadian Neurological Society \\ Société canadienne de neurologie \\ Canadian Society of Electroencephalographers, Electromyographers and \\ Clinical Neurophysiologists \\ Société canadienne des électroencéphalographistes, électromyographistes \\ et neurophysiologistes cliniques \\ Canadian Association of Neurological and Neurosurgical Nurses \\ Association canadienne des infirmières en neurologie et neurochirurgie
}

OFFICERS OF THE CONGRESS/OFFICIERS DU CONGRES
$\begin{array}{lll}\text { President } & \text { William Lougheed } & \text { Président } \\ \text { Director } & \text { Frederick Andermann } & \text { Directeur }\end{array}$

\section{SCIENTIFIC PROGRAM/PROGRAMME SCIENTIFIQUE}

$\begin{array}{lll}\text { Chairman } & \text { Francis E. LeBlanc } & \text { Président } \\ \text { R. G. Lee } & & \text { K. M. Hunter } \\ \text { A. K. Brownell } & & \text { T. P. Seland } \\ \text { R. H. Haslam } & & \text { S. T. Myles }\end{array}$

LOCAL ARRANGEMENTS/DISPOSITIONS LOCALES

$\begin{array}{lll}\text { Chairman } & \text { Ian M. Turnbull } & \text { Président } \\ \text { A. R. Anzarut } & \text { H.G. Dunn } & \text { P. D. Moyes } \\ \text { K. Berry } & \text { D. E. Griesdale } & \text { C. A. Simpson } \\ \text { P. J. Bratty } & \text { M. D. Low } & \text { V. P. Sweeney } \\ \text { C. L. Dolman } & & \text { G. B. Thompson }\end{array}$

\section{LADIES' COMMITTEE/COMITE FEMININ}

$\begin{array}{lll}\text { Chairman } & \text { Janis F. Turnbull } & \text { Présidente } \\ \text { Sheila Anzarut } & \text { Barbara Griesdale } & \text { Pat Moyes } \\ \text { Sally Berry } & \text { Terry Hashimoto } & \text { Mary Sweeney } \\ \text { Elizabeth Bratty } & \text { Celia Low } & \text { Sally Thompson }\end{array}$

\section{NURSES' PROGRAM/PROGRAMME DES INFIRMIERES}

Chairman

Donna Brett

Barbara Fergie

Présidente

Elaine Sleath

Sue Goode

Maureen Worabec

\author{
CANADIAN NEUROLOGICAL SOCIETY \\ SOCIETE CANADIENNE DE NEUR OLOGIE \\ President \\ Past President \\ Vice-President \\ Secretary-Treasurer \\ F. Andermann \\ C. Belanger \\ J. R. Wherrett \\ R. G. Lee
}

Président

Ex-Président

Vice-président

Secrétaire-trésorier

\section{COUNCILLORS/CONSEILLERS}
A. J. Aguayo
M. J. Newman
H. G. Dunn
D. W. Paty
J. B. Martin
D. Simard

CANADIAN SOCIETY OF ELECTROENCEPHALOGRAPHERS, ELECTROMYOGRAPHERS AND CLINICAL

NEUROPHYSIOLOGISTS

SOCIETE CANADIENNE DES

ELECTROENCEPHALOGR APHISTES,

ELECTROMYOGRAPHISTES ET NEUROPHYSIOLOGISTES CLINIQUES

$\begin{array}{lll}\text { President } & \text { M. Brandstater } & \text { Président } \\ \text { Past President } & \text { K. Metrakos } & \text { Ex-président } \\ \text { Secretary-Treasurer } & \text { J. Reiher } & \text { Secrétaire-trésorier }\end{array}$

COUNCILLORS/CONSEILLERS
C. Bolton
N. Giard
R. Broughton
J. G. Humphrey

A. Eisen

\begin{tabular}{|c|c|c|}
\hline \multicolumn{3}{|c|}{$\begin{array}{l}\text { CANADIAN ASSOCIATION OF NEUROLOGICAL AND } \\
\text { NEUROSURGICALNURSES }\end{array}$} \\
\hline \multicolumn{3}{|c|}{$\begin{array}{l}\text { ASSOCIATION CANADIENNE DES INFIRMIERES EN } \\
\text { NEUROLOGIE ET NEUROCHIRURGIE }\end{array}$} \\
\hline $\begin{array}{l}\text { President } \\
\text { Past President } \\
\text { President Elect-Sec. } \\
\text { Treasurer }\end{array}$ & $\begin{array}{l}\text { Beth Cook } \\
\text { Betty King } \\
\text { Pauline Weldon } \\
\text { Judy Conrad }\end{array}$ & $\begin{array}{l}\text { Présidente } \\
\text { Ex-Présidente } \\
\text { Présidente élue-Secrétaire } \\
\text { Trésorier }\end{array}$ \\
\hline
\end{tabular}

\section{DISTINGUISHED GUESTS OF THE XIII CONGRESS INVITES DU XIII CONGRES}

Dr. Frank Turnbull, Associate Clinical Professor (Surgery), University of British Columbia, Vancouver.

"Goose Quills \& Burr Holes"

SOCIETE CANADIENNE DE NEUROCHIRURGIE

$\begin{array}{lll}\text { President } & \text { W.M. Lougheed } & \text { Président } \\ \text { Past President } & \text { J.C. Giroux } & \text { Ex-président } \\ \text { Vice-president } & \text { J. F. R. Fleming } & \text { Vice-président } \\ \text { Secretary-Treasurer } & \text { R.G. Elgie } & \text { Secrétaire-trésorier }\end{array}$

Secretary-Treasurer R. G. Elgie Secrétaire-trésorier

\section{COUNCILLORS/CONSEILLERS}
F. Durity
J. Francoeur
K. M. Hunter
M.T. Richard
A. R. Hudson
N. A. Russell

Dr. George Austin, Professor and Chief of the Section of Neurosurgery, Loma Linda University School of Medicine, Loma Linda, California. "The Problems and Future of Stroke Prevention"

Dr. John O'Brien, Professor and Chairman, Department of Neurosciences, School of Medicine, University of California at San Diego.

"Current Advances in Neuronal Storage Diseases" 


\section{CEREBR OVASCULAR I (Plenary Session)}

\section{Focal Cerebral Ischemia and Reperfusion - The Golden Period}

Richard A. R. Fraser, Shinichi Yoshida and Russel Patterson, New York

Amongst the crucial questions about cerebral ischemia that remain to be answered are (1) what is the duration of ischemia necessary to produce neuronal death and (2) can this time period be extended, so that attempts at cerebral reperfusion can be accomplished within the time limits necessary for return of useful cerebral function. (3) What is the role of "no reflow" or microvascular obstruction in cerebral ischemia.

Fifty-four rhesus monkeys were used. Experiments were performed utilizing microsurgical transorbital exposure of the intracranial carotid artery bifurcation. Animals were subjected to MCA clipping into 4 groups of 12 , plus 6 additional animal controls $-12-1$ hour, 12-3 hours, 12-6 hours. 12-permanent ( 3 weeks).

One half of each group of 12 were treated with a vasopressor in order to keep systemic blood pressure 25 torr above normal for the duration of clip application. After clip removal animals were examined daily till sacrifice - 3 weeks later. After formalin-carbon black perfusion, pathological examinations were carried out upon the serially sectioned brains.

Data from these experiments reveal that focal cerebral ischemia for 1 and 3 hours is well tolerated. These results closely parallel the pathological outcome. A drastic change occurs with longer ischemia - 6 hours of MCA occlusion is virtually identical to permanent occlusion. Hypertension is clearly of benefit up to 3 hours. After this no effect, or a deleterious one is revealed. Microvascular obstruction was rare before 6 hours but frequent in all the permanently clipped animals.

These data support the notion that focal cerebral ischemia can be tolerated for periods sufficient to warrant attempts at repufusion for a short time only. Hypertension reduces the minor morbidity during this period but does not extend the viable reperfusion period. These data also suggest that "no reflow" microvascular obstruction does not contribute to acute infarction as it occurs after neuronal death has already taken place.

\section{Treatment of Acute Focal Ischemia with Mannitol and High-Dose Dexamethasone: An Experimental Investigation in Cats} John R. Little, Montreal

The effects of mannitol and high-dose dexamethasone upon focal cerebral ischemia were studied in conscious cats following middle cerebral artery (MCA) occlusion. A simple implanted device was applied to the poximal segment of the left MCA of 50 adult cats. The artery was occluded 3 or 4 days following recovery from the initial procedure. In the acute experiments, 10 cats received no treatment and 10 cats were given mannitol ( 1.2 $\mathrm{gm} / \mathrm{kg} \mathrm{IV).} \mathrm{The} \mathrm{initial} \mathrm{neurological} \mathrm{response} \mathrm{was} \mathrm{similar} \mathrm{in} \mathrm{both} \mathrm{groups,}$ that is, agitation, forced circling, and right hemiparesis. The cats in the treated group improved clinically between 3 and 6 hours. Perfusion with a mixture of colloidal carbon and buffered paraformaldehyde was carried out from 30 minutes to 12 hours following MCA occlusion. The results of the morphological examination of the brains indicated that mannitol had a transient protective effect upon the cerebral tissue during the initial 6 to 12 hours of ischemia. This beneficial effect was attributed partly to prevention of capillary narrowing and suppression of ischemic edema. In the subacute experiments, 10 cats received no treatment, 10 cats were given mannitol and 10 cats were given mannitol and high-dose dexamethasone. Although early transient clinical improvement occurred in most of the treated animals, morphological findings in the treated and untreated cats following 48 hours of ischemia were not significantly different. Despite the finding that infarct size at 48 hours was not reduced, however, the delay in the development of irreversible changes by treatment with mannitol alone was thought to be important as it may allow a longer period of time for the institution of more definitive forms of therapy.

\section{The Acute Stroke: A View from an Intensive Care Stroke Unit}

John W. Norris and Vladimir Hachiniski, Toronto

Three hundred and sixty patients were admitted to an intensive care stroke unit, and after investigation, 72 proved not to have had strokes. Continuous observation indicated that $54 \%$ of the misdiagnosed cases actually had Todd's paralysis and other post-epileptic states. Subdural hematomas and other mass lesions accounted for fewer than $5 \%$ of the misdiagnoses. No cardiac cases were misdiagnosed as stroke.
Continuous ECG monitoring showed that $50 \%$ of 312 patients with cerebral infarct or hemorrhage had cardiac arrhythias compared to controls ( $p$ less than 0.001). Arrhythias were more common with hemispheric than with brain stem lesions ( $\mathrm{p}$ less than 0.05 ). Closer study of 64 patients showed that 11 (17\%) had elevated serum CPK-MB (cardiac) isoenzyme levels. Three patients showed cardiac myocytolysis at autopsy, suggesting that the brain lesion may affect cardiac function and perhaps prognosis.

Routine EEG monitoring is of limited value as a diagnostic tool but prolonged sleep EEG recordings were found to relate closely to the extent of the underlying cerebral pathology. Patients with maximal disturbance of sleep EEG patterns, have the worst outlook. The correct prognosis was predicted in 33 of 35 patients by serial sleep EEG recordings.

With modern diagnostic facilities, stuctural lesions such as subdural hematoma and tumors no longer represent a problem in differential diagnosis but post-ictal states especially Todd's paralysis are often confused with stroke. ECG and isoenzyme monitoring suggest that myocardial disfunction may be a cause as well as a consequence of an acute cerebral lesion. Monitoring of the sleep EEG can add information useful in the assessment and prognosis of the acute stroke.

\section{Results of Extracranial-Intracranial Artery Anastomosis (EC-IC) in Stroke Patients \\ H. Schutz, F. Taylor, K GerBrugge and M. Chiu, Toronto}

The results of EC-IC anastomosis were analyzed in our first 25 patients (10 with T.I.A.s, 9 with RINDs, 6 with moderate infarctions.). The mean age was 56 years. The average time between onset of stroke and surgical procedure was five months. The average duration of follow-up was thirteen months. Angiography showed 16 internal carotid artery occlusions with lesions in tandem and 9 middle cerebral artery or carotid siphon occlusions or stenoses. Region cerebral blood flow (rCBF) was measured pre- and post-operatively in 23 patients using intracarotid injection of Xenon 133. Wash-out curve analysis from 8 selected regions of the brain was done "one line" according to the method of Lassen and Ingvar. Focal reductions of regional cerebral flood flow (less than $30 \mathrm{ml}$. per $100 \mathrm{Gm}$. per minute) in conjunction with a clinical and radiological criteria were the principal indications for surgery. Surgical expertise in microvascular end-to-side anastomoses was gained in the laboratory, utilizing femoral artery $(0.7$ $\mathrm{mm}$.) to vein $(1.0 \mathrm{~mm}$.) anastomosis in over 50 rats with a patency rate of $100 \%$.

There was no operative mortality. Wound healing was delayed in five patients. One major stroke occurred within six hours of surgery due to the occlusion of a severely stenosed middle cerebral artery with non-patency of the anastomosis. An $80 \%$ patency rate at 3-9 months post-operatively was obtained in the last twelve patients. During the follow-up period, no patient suffered recurrent cerebral infarction and two patients experienced a single T.I.A. Neurological deficits remained unchanged in 19 patients and improved in 6. Regional cerebral blood flow improved dramatically (50 $100 \%$ increase) in those patients where the anastomosis was able to assume the entire load of the middle cerebral artery.

The results suggested this procedure may reduce stroke recurrence and improve cerebral blood flow in these revascularized patients.

\section{The Canadian Cooperative Platelet-Inhibition Drug Trial H. J. M. Barnett, London}

Twenty-four hospitals in 12 cities cooperated to enter 585 patients with TIA or partial non-progressive stroke into a randomized trial to determine the efficacy of ASA or sulfinpyrazone in preventing stroke or death. The patients were treated for an average of 717 days, a $99 \%$ follow-up was achieved and patients were assessed up to the end of the study, 30 June, 1977 , or until death.

A review committee considered the signs and symptoms of all patients entered and the exclusion of doubtful cases was strict. Angiography was carried out in $76 \%$ of the patients. Among all the carotid cases entered, $78 \%$ had lesions appropriate to the symptoms. This was true of $82 \%$ of the vertebral-basilar cases and $78 \%$ of those with symptoms in both carotid and vertebral-basilar territory. The severity of the arterial lesions varied somewhat from centre to centre but was acceptably uniform. In the largest centre, with 118 carotid cases entered, $58 \%$ had lesions that would have been considered "surgical", and 5\% more were "probably surgical", by the most usual criteria. Thirteen percent had lesions that were appropriate but inaccessible or occlusive, while $21 \%$ had minimal disease or normal arteries. Three percent were not studied angiographically. 
The results of the study determined that in this group of 585 cases, asprin effectively reduced stroke or death in males. Neither drug was effective in fermales and sulfinpyrazone was ineffective in both. No antagonism or synergism was detected between the two treatment modalities. The significance of the response to asprin was diminished if patients were diabetic or had previous history of myocardial infarction. No study of platelet-inhibiting drugs that has been completed has shown, as yet, a significant response in females.

\section{CEREBROVASCULAR II}

\section{Usefulness of Krypton-77 Positron Emission Tomography in Stroke}

\section{Patients}

Y. L. Yamamoto, J. Little and W. Feindel, Montreal

A circular positron detecting device, using the non-invasive Krypton-77 inhalation technique, is capable of measuring quantitative and true topographical regional cerebral blood flow ( $\mathrm{rCBF}$ ) in a cross section of the head. We have used this technique to evaluate topographical changes of $\mathrm{rCBF}$, before and after medical and surgical treatments, in over 80 patients with occlusive cerebrovascular disease. CT scan and dynamic cerebral perfusion study with a gamma scintillation camera were performed in all cases. Direct measurement of $\mathrm{rCBF}$ in the exposed brain was also performed, using the Xe-133 clearance technique with four semiconductor detectors, before and after by-pass surgery in 10 patients. Our findings indicate that the moderately reduced rCFB values of 15 to $35 \mathrm{ml} / 100$ $\mathrm{g} / \mathrm{min}$, shown in the Krypton-77 P.E.T. study, are often correlated with moderate to severe reduction of the cerebral dynamic study, but no abnormality is apparent in the CT scans. The focal lower density in the CT scans, due to focal cerebral infarction, received a minimal amount of Krypton-77 gas with very low rCBF values of 0 to $9 \mathrm{ml} / 100 \mathrm{~g} / \mathrm{min}$.

The results of our studies indicate that improvement of rCFB in the cortical and subcortical ischemic lesions are extensive and of a higher magnitude after by-pass surgery than that achieved with medical therapy with inhalation of $5 \% \mathrm{CO}_{2}$.

\section{Outcome of Cerebral Infarction - A Canadian Experience}

M. Freedman, J. W. Norris and V. C. Hachinski, Toronto

The outcome of 290 patients with cerebral infarction admitted to an intensive care stroke unit in a general hospital were followed for up to two years.

There were 240 patients with hemispheric infarction and 50 with brain stem infarction. One hundred and seventy-eight $(61 \%)$ had hypertension, $64(22 \%)$ had diabetes, and $222(75 \%)$ had heart disease.

Forty-five $(16 \%)$ patients died during their first admission and a further $37(13 \%)$ after one year. After two years, $13(13 \%)$ of the remaining patients followed up have died.

The degree of disability was graded and after one year, $57(20 \%)$ cases were functionally normal, $90(31 \%)$ were mildly disabled, $31(11 \%)$ were semiambulant and $8(3 \%)$ were totally incapacitated. The remainder were lost to follow-up. A similar distribution of morbidity was seen after two years. The annual incidence of further strokes was $11 \%$ and of seizures was $5 \%$. Seizures developed only in those patients with hemispheric infarction.

Although patients with cerebral infarction remain at risk from further strokes and seizures, the majority attain self sufficiency in activities of daily living by one year.

\section{A Randomised Trial of Sulfinpyrazone in Patients Undergoing Carotid Endarterectomy for Threatened Stroke}

The Canadian Cooperative Study Group (F. E. LeBlanc)

One hundred and twelve patients referred to five Canadian centres with threatened stroke underwent carotid endarterectomy. Most of these patients met the criteria of entry to the Canadian Cooperative Study of Recent Recurrent Presumed Cerebral Emboli (RRPCE). All had angiographic studies which demonstrated a high-grade stenosis or occlusion at the origin of the internal carotid artery in the neck. In one hundred and one of these, the side appropriate to reported or observed episodes of transient cerebral ischemia was involved. Post-operatively, these patients entered a drug trial where they were randomly placed on either sulfinpyrazone or a placebo. Patients were followed for twenty-one months, $95 \%$ of which time they were on the study drugs and complete follow-up achieved in $98.5 \%$.
The study drug (sulfinpyrazone) in this surgical series of patients with threatened stroke, did NOT significantly reduce the risk of continuing transient ischemic attacks (TIAs), stroke or death.

In the study period, four patients had strokes ( $2.0 \%$ per year), three of these involving the distribution of the endarterectomised vessel. Seven patients died (3.6\% per year) only one of these from a stroke. Thus the incidence of stroke or death in this series was $5.6 \%$ per year. Twenty-eight patients had one or more TIAs following surgery (14\% per year). The incidence of TIA, stroke or death following carotid endarterectomy in this study was approximately $20 \%$ per year.

\section{Carotid Endarterectomy: A Follow-up of 150 Consecutive Surgical Cases and 120 Non-Operated Cases of Cerebrovascular Disease \\ V. P. Sweeney and X. Fornazzari, Vancouver}

A retrospective study measured the morbidity, mortality and functional status of all patients treated by carotid endarterectomy at the Vancouver General Hospital in the years 1971 through 1975. The subsequent course of 150 surgical cases was compared with that of 120 patients similarly investigated for cerebrovascular disease who also had demonstrable cervicocranial lesions but who were not treated surgically. Personal examination of patients was carried out when possible.

Sixty-nine patients had died; 28 (15 cardiac, 6 stroke) in the surgical group and 41 (17 cardiac, 15 stroke) in the non-surgical group. Non-fatal strokes occurred in 8 of the surgical group and in 5 of the non-surgical group. Equal numbers (35) in each group experienced further transient ischemic attacks. The functional status of survivors in each group was comparable.

Further cardiovascular events appeared to be related to a number of clinical variables but pre-existing cardiac disease and the extent of cerebrovascular involvement were the main determinents. Careful selection of cases for surgery is essential to improve the incidence of fatal and nonfatal cardiovascular events.

\section{The "Routine" Use of Intraluminal Shunting in Carotid Endarterectomy Brien G. Benoit and Nosrat L. Nabavi, Ottawa}

This study, ongoing since 1972, analyzes over 160 endarterectomies carried out under the direction of a single surgeon, thereby ensuring standardization of technique.

Only 15 patients were operated upon without a shunt. There was one death, a patient operated on in the acute phase. Postoperative stroke occurred in one patient, in whom a shunt could not be inserted because of tortuosity of the internal carotid artery.

An intraluminal shunt was used in 148 cases, and in all patients undergoing surgery during the past 3 years. Three patients died in the immediate postoperative phase. One suffered a massive myocardial infarction at the end of the procedure, another died of cardiac tamponade following a combined carotid endarterectomy and aorto-coronary bypass procedure, and one patient with known malignant disease, died of his cancer some weeks after awakening from the endarterectomy with an increased neurological deficit.

Two patients awoke with increased neurological deficits, following endarterectomy. Both had a previous cerebral infarction, with deficit. No patient, operated upon for transient ischemic attacks, suffered a neurological deficit following endarterectomy. In this series of shunted patients, the mortality was $2 \%$, and the surgical morbidity was also $2 \%$. This compares favorably, with other published series, in which the mortality ranges from 0.7 to $7 \%$, and the morbidity from 1.5 to $10 \%$. Most of our patients were in the ASA categories II or III.

The average occlusion time during introduction of the shunt, was 1.5 minutes ( 100 seconds), and the time of occlusion during decannulation was 2.3 minutes (143 seconds). The average surgical time was slightly over 2 hours (123 minutes). The shunt used in all operations was a polyvinyl chloride catheter ("USCI Carotid Bypass Tube") with a 10 or $12 \mathrm{~F}$ size.

In summary, our experience with an intraluminal shunt, would indicate that it is a safe technique, which can be used routinely. It affords the advantages of allowing for an unhurried and meticulous endarterectomy, during which the brain is being adequately perfused. In addition, the closure of the arteriotomy in small vessels, is facilitated by the stent effects of the tube, which prevent undue narrowing of the vessel. We were unable to insert a shunt, in only one case, and have not found increased difficulty in performing an endarterectomy, because of the shunt. Details of the technique will be described. 
The "shunt, no shunt", controversy will undoubtedly continue, but if the anaesthesia-surgical team becomes practiced in the routine use of an indwelling shunt, most of the disadvantages of the technique, are easily eliminated.

\section{Carotid Endarterectomy: Is an Internal Shunt Necessary?}

G. G. Ferguson, W. T. Blume, K. McNeill and G. P. Varkey, London

The necessity for routine or frequent use of an internal shunt during carotid endarterectomy is controversial. Sundt et al (J. Neurosurg 41: 310 320,1974 ) assume a high incidence of clinically significant ischemia during cross-clamping for carotid endarterectomy, and advocate the routine use of a shunt in all cases in which "significant" changes in the intra-operative E.E.G. occur. As a result they used a shunt in $50 \%$ of their 113 cases, and ascribe a low rate of new post-operative ischemic deficits to this practice.

This series consists of 54 consecutive carotid endarterectomies performed by one surgeon. An internal shunt was used only twice, early in the series. Of the 54 patients, only one had a new post-operative deficit. This was minor and nearly complete recovery occurred. In the last 17 cases continuous intra-operative E.E.G. recordings have been made. In 7 of these there were "significant" E.E.G. changes according to the Mayo criteria (suppression or delta activity). In spite of these changes, and in spite of the fact that this was a relatively "high risk" group of patients ( 4 had contralateral carotid occlusion and 3 severe contralateral carotid stenosis), a shunt was not used in any of these 17 cases. There were no new deficits post-operatively.

These results raise some doubt as to the Mayo Clinic assumptions and conclusions. From our experience it appears that "apparent" ischemia during carotid cross-clamping is well tolerated in the controlled operating room situation, and that the use of an internal shunt is rarely, if ever, required during carotid endarterectomy.

\section{The Role of Surgery in the Treatment of Spontaneous Intracerebral Haemorrhage}

M. Besser and H. W. K. Barr, London

A retrospective review of 58 non-randomized patients with primary intra-cerebral hematomas seen over the last seven years at Victoria Hospital, London, Ontario, were analysed to assess the value of surgical therapy. Excluded were haemorrhages secondary to tumors, trauma aneurysms, A.V.M.'s and those occurring in the brainstem or cerebellum. The haematoma in each patient was localized by either angiography or C.T. Scan.

One-third of the patients were not hypertensive. The overall mortality rate was $59 \%$. Twenty-three patients were treated surgically (average age 53.5 years) with a mortality rate of $48 \%$. Patients treated conservatively had a mortality rate of $66 \%$ (average age 56.5 years). Although patient selection prevents direct comparison of the two groups, interesting information has been derived and with the accuracy of the C.T. Scanner, may direct future treatment in our centre.

Patients operated upon two or more days following the ictus (average time interval 8.8 days) had a mortality rate of $15 \%$ compared to a $90 \%$ mortality rate if surgery was performed within 48 hours of the ictus. The level of consciousness greatly influenced the prognosis. If the patient was unconscious at the time of surgery the mortality rate was $90 \%$, whereas the mortality rate fell to $15 \%$ in conscious patients. Those conscious patients treated conservatively had a mortality rate of $20 \%$; however, the quality of survival improved in this group with surgery $(63 \%$ independent compared to $37.5 \%$ ). All patients who were unconscious at the time of surgery and who survived remained either dependent with major deficit or in a vegetative state.

Review of this series would seem to indicate that patients who are unconscious with deep haemorrhages do not benefit from surgery. If the patient is conscious, surgical mortality is low and the quality of survival superior when compared to conservative therapy.

\section{Superficial Temporal Artery to Middle Cerebral Artery Anastomosis: A Pathologic Study of Two Cases \\ P. Steinbok, K. Berry and C. L. Dolman, Vancouver}

Superficial temporal artery to middle cerebral artery (STA-MCA) anastomoses were examined at autopsy in two patients who survived two years and five years respectively after the cerebral revascularization procedure.
In each patient transient ischemic attacks ceased in temporal relation to STA-MCA anastomosis performed on the side of the cerebral dysfunction, and neither patient had further neurologic symptoms up to the time of death from myocardial infarction.

Identification of the actual anastomotic sites at autopsy was rendered impossible because of marked fibrosis in the region of the surgery. However in both patients intimal fibrosis and medial damage were noted in the superficial temporal artery immediately adjacent to the anastomosis. In the patient surviving two years, the lumen of the superficial temporal artery was only slightly compromised by these changes, but in the patient surviving five years, the artery was at least $96 \%$ occluded. The middle cerebral arteries adjacent to the anastomosis were widely patent and showed focal areas of intimal hyperplasia with no abnormalities of the media.

Pathologic study of the bypass in such cases should provide further helpful information in assessing the indications and response to this form of treatment.

\section{CHILD NEUROLOGY}

Behavioural and Cognitive Effects of Daily Phenobarbital in Toddlers (A Double-Blind Placebo Controlled Study)

Carol S. Camfield, Sheila Chaplin, Anna Beth Doyle, Carl Cummings, and Peter R. Camfield, Montreal and Halifax

A randomized prospective doubleblind placebo controlled study of the efficacy of single daily dose of Phenobarbital $(\emptyset)$ to prevent recurrent febrile seizures permitted objective assessment of behavioural and cognitive effects of $\emptyset$ in toddlers. There were no significant differences in global I.Q. (Binet or Bayley Scales) between placebo $(n=14)$ and $\emptyset(n=24)$ groups after 8 to 12 months of medication. However, blood plasma level of $\theta$ correlated negatively with Binet memory test scores, $r=-.52$, $p$ less than .01 . In other words, the higher the $\theta$ level the lower the memory score. Hyperactivity was not seen. The only behavioural changes noted were increased fussiness and a special sleep disturbance consisting of awakening several hours at night. $4 / 65$ children were withdrawn from the study because of these unacceptable changes ( 30,1 placebo). $4 / 35$ children on $\emptyset$ had consistent dose-related behavioural changes at $5 \mathrm{mg} / \mathrm{kg} / \mathrm{d}$ which disappeared at $2-3 \mathrm{mg} / \mathrm{kg} / \mathrm{d}$ with most serum levels remaining greater than $10 \mathrm{mg} \%$. Transient behavioural changes (less than 4 week) were seen equally in both groups $(7 / 30$ on placebo and $8 / 35$ on $\varnothing$. After 12 months in this study, most parents could not distinguish between drug and placebo. Only the parents of the 4 children with dose-related effects guessed Phenobarbital correctly. In conclusion, the behavioural effects of $\emptyset$ are varied, including unacceptable, transient, and dose related. Effects of $\phi$ on memory are definite, however, of uncertain significance as the global I.Q. is unchanged. If the drug is indicated, serum levels should be maintained at the lowest effective level to minimize effects on memory.

\section{Giant Axonal Neuropathy (GAN):}

A disorder of the central and peripheral nervous system: clinical, neurophysiological, and ultra-structural findings in two patients and review of five previous cases

Gordon Watters, George Karpati, Stirling Carpenter, Peter Humphreys and Fred Andermann, Montreal

In 1972 the first patient with giant axonal neuropathy (GAN) was reported and to the present there have been four other well documented cases, one of which was from our center. We are adding two new patients with a review of the disorder, as it manifests in these 7 patients.

Consanguinity occurred in three of the 7 families. There was a total of twelve siblings and all were normal.

All seven patients, 3 girls, 4 boys, were born after full term normal pregnancies. Five walked before the age 15 months; while one walked at 18 months; and the other at 3 years. In the five in which height was noted, four were below the 3rd percentile, while all had normal head circumferences. All seven had unusual kinky, curly hair from early in life, unique in their families. Intelligence was normal or low normal in all.

Onset of symptoms was between 2 and 3 years in five of the seven, and presented as difficulty with walking and increased falling. A broad based gait with planovalgus feet was soon noted, a hallmark of the disorder. Ptosis and slurred speech occurred in four. Stretch reflexes were absent in most as early as 2 years. A slowly progressive weakness of legs developed. Arms were also affected but less severely. There was generalized hypotonia and in the legs, distal wasting. Peripheral sensory loss to touch was noted 
in six, and to vibration and position in three. At age 5 to 6 years vertical and horizontal nystagmus appeared. Ability to walk has been retained until age 10 years at least.

In all CSF was normal as were serum CPKs. EEGs showed mostly mild abnormalities. A pneumo-encephalogram in one, a vertriculogram in another and $\mathrm{CT}$ scans in two patients were normal.

Of special note EMG and nerve conduction studies were normal in the first 3 years even when deficits were present, but by age 4 or 5 years these studies showed an axonal degenerating neuropathy more marked in the legs. Sensory action potentials were absent at this stage. Sural nerve biopsies in all, the earliest at age 3 years, showed greatly enlarged axons packed with neurofilaments, typical of the disease.

The ascertainment of three unrelated patients with GAN from one center suggests this is a relatively common disorder with a distinct recognizable clinical presentation. Further, these observations indicate that GAN is a heritable metabolic disorder affecting the central and peripheral nervous system, hair structure, and perhaps somatic growth, with the basis of the disorder to be elucidated.

\section{Observations on Ocular Myasthenia Gravis in Ten Children \\ N. Barnor and D. McGreal, Toronto}

Myasthenia gravis is now accepted as an immunological disease involving the acetylocholine receptors at the neuromuscular junction. In children, three clinical, (neonatal, congentital and juvenile) forms have been recognized. In the past twenty-two years, twenty-five children have had a diagnosis of myasthenia gravis made. Of these, ten children presented with purely ocular signs. The clinical courses of these children is reviewed.

Two of these children had no clinical symptoms eight and ten months after diagnosis and they remain asymptomatic; in the one case, eleven years later and, in the other, eighteen months later. One boy developed diabetes mellitus nine years after diagnosis and eight years after resolution of the myasthenia gravis. Two boys developed generalized disease, one boy developed bulbar involvement four months after diagnosis which responded to anticholine-esterase treatment which was discontinued five years from diagnosis. The other boy had two relapses, five years apart, being asymptomatic in between, then developed generalized myasthenia gravis over a period of four months. Three patients are still on medication. These findings suggest that ocular myasthenia gravis in children is usually a benign condition.

\section{The "Encysted" Cisterna Magna In Children}

\section{Archer and H. Darwish, St. Louis and Calgary}

A large cisterna magna is often a "normal variant" in childhood. It is also reported commonly in association with cerebellar hypoplasia and corpus callosal agenesis, where a compensatory filling mechanism is suggested. Juhl and Wesenberg had reported that the area under lambdoinnominate line was increased in the Dandy-Walker syndrome, reflecting expansion of the posterior fossa. This was a subjective assessment, and standard area measurements were not developed. Standardized assessment is important since the bony landmarks reflecting the position of the torcular hereophili and the transverse sinuses may not be seen under 2 years of age. We used Polar planimetry, as well as computerized techniques to assess the area under the lambdo innominate line on routine skull films. A ratio expressing the relation of this area to total skull area at different ages in infancy and early childhood was developed.

We report three children who had enlarged cisterna magna determined by computed tomography of the skull, pneumoencephalogram, and radionuclide cisternography. Macrocephaly was present in two. Corpus callosal agenesis and cerebellar hypoplasia were associated with enlarged cisterna magna in two cases. The posterior fossa was expanded, as determined by area measurements on skull roentenograms, and elevation of the torcular herophili on radionuclide angiography. In one case, macrocephaly was progressive in spite of functional ventricular shunting procedures. Shunting of the "cisterna magna" was then performed.

We suggest that the enlarged cisterna magna in these children is functionally encysted, performing as a dynamic space occupying lesion in spite of evidence of communication on pneumoencephalography. Early evaluation of routine skull roentgenograms using the posterior fossa area ratio may be useful to detect this anomaly.

\section{NEUROMUSCULAR I}

Muscle Disease with Exercise Pain and Abundant Tubular Aggregates: A Distinct Clinico-Pathological Entity.

George Karpati and Stirling Carpenter, Montreal

A 60 year old man complained of increasing pain in the buttocks and thighs on moderate exercise for 2 months. Examination was normal except for slight weakness of hip flexors and glutei and absent knee jerks. He had mild, maturity-onset diabetes mellitus without evidence of vascular insufficiency or other complications. Biopsies of both quadriceps muscles showed abundant, large tubular aggregates in type 2 fibers. A forearm metabolic study showed no defect in lactate generation, oxygen and glucose uptake and ionic fluxes of muscle on exercise. His symptoms responded excellently to oral intake of Dantrolene Sodium.

Another 50 year old man complained of annoying pain in his shoulder girdle and proximal arm muscles on exercise for 8 months. His deltoid biopsy showed findings similar to the first patient.

On the basis of the experience with these patients and a few other ones in the literature, abundant tubular aggregates with exercise pain appear to be a clinically distinct syndrome even though occasional tubular aggregates may occur in different neuromuscular diseases. The pathophysiology of the syndrome remains elusive, but symptomatic treatment with Dantrolene seems effective.

\section{Insulin Receptor Binding and Carbohydrate Metabolism in Myotonia Dystrophica}

\section{A. J. Hudson, G. J. M. Tevaarwerk, K. P. Strickland and C. J. Lin,} London

Amongst the more prominent biochemical abnormalities of myotonia dystrophica (MD) are glucose intolerance, hyper-insulinism and resistance to exogenous insulin. In a study of insulin resistance, curculating mononuclear cells were obtained from $12 \mathrm{MD}$ patients and 12 matched normal control subjects and incubated with $50 \mathrm{pg} 1^{-125}$ monocomponent insulin alone and in the presence of gradually increasing amounts $(0.005 \mu \mathrm{g}$, $0.01 \mu \mathrm{g}$ and $0.025 \mu \mathrm{g}$ of unlabeled insulin. Binding in the two groups was $0.65 \pm 0.1 \%$ (mean \pm S.E.) in MD and $1.26 \pm 0.08 \%$ in the normal group per $10^{6}$ monocytes. The addition of unlabeled insulin produced a parallel decrease in binding in the two groups e.g. $0.35 \pm 0.04 \%$ in the MD cases and $0.84 \pm 0.13 \%$ in the normal subjects with $0.005 \mu \mathrm{g}$ added insulin. Scatchard analysis of the results suggest that reduced insulin binding in MD is due to a decreased number of receptors rather than an alteration of affinity. No difference in the effect of normal and MD is due to a decreased number of receptors rather than an alteration of affinity. No difference in the effect of normal and MD sera on insulin binding to cultures human lymphocytes (IM9 cells) was found indicating that reduced binding is not likely due to receptor blockade.

A negative correlation was found between basal insulin levels and mononuclear cell insulin binding in normal subjects. However, no such correlation exists for the MD group. On the other hand, a positive correlation was observed between the reduced level of insulin binding to monocytes and the decreased fall in the plasma glucose in response to exogenous insulin. It is concluded that a primary cellular defect is likely responsible for the reduced insulin binding and impaired glucose assimilation observed in MD patients.

Incorporation of ${ }^{3} \mathrm{H}(\mathrm{G})$ L-Leucine into Single Muscle Fibers in Duchenne Dystrophy

George Monckton and Halyna Marusyk, Edmonton

Observations of increased protein synthesis have been made for a number of years in Duchenne muscular dystrophy. A search for an underlying cause for the increase has not been successful, and opinions as to the causation have been varied. Some authors have favoured the conclusion that increased uptake of isotopes is related to the basic genetic defect in a direct way. Others have considered the increased protein synthesis and isotope uptake to be due to regenerative efforts in the muscle evoked by muscle damage.

The method of single muscle fiber autoradiographic study with the uptake of isotopes enables an accurate assessment of the level of incorporation of amino acid per unit area/volume of a muscle fiber. When we applied this method in biopsy specimens of patients with Duchenne dystrophy our results showed a markedly increased level of uptake of ${ }^{3} \mathrm{H}(\mathrm{G}) \mathrm{L}$-leucine in 
the smaller muscle fibers, whereas the larger fibers show a more normal level of uptake. We feel that these findings of increased uptake in the smaller fibers is more consistent with intensive regenerative efforts by previously damaged muscle than a direct result of an intrinsic defect of protein synthesis.

\section{Muscle Biochemical Defects in Chronic Alcoholics One Day and Two Weeks After the Last Drink. \\ P. L. Carlen, L. A. Huszar, R. B. Rewcastle, G. Modell, and B. Kapur, Toronto}

Chronic alcoholics often show proximal weakness which can recover markedly over days to weeks with maintained abstinence. This study attempted to examine underlying mechanisms of alcoholic muscle weakness.

Repeated muscle biopsies were performed on chronic alcoholics: within 24 hours of the last drink and 2 weeks later. Ischemic lactate tests were done on the first, third, seventh, tenth and fifteenth day of abstinence. Five of eight patients showed depressed lactate generation after 1 minute of ischemic exercise. Three of the five showed an increase in muscle strength which correlated with improved ischemic lactate tests. The other two patients showed no clinical weakness with close to normal ischemic lactate tests. Biochemical determinations have shown that the initial phosphorylase activity was within normal limits, but this activity usually increased by $50 \%, 2$ weeks later. Some patients showed no in vitro lactate generation using their first muscle sample with glycogen substrate. Possible explanations of the above data are: a defect of muscle phosphorylase activation or a depletion of muscle glycogen stores. Pathological examination of the muscle showed non-specific abnormalities not correlated with the biochemical changes. Electromyographic studies done on four selected patients showed no abnormality.

\section{NEUROBIOLOGY I}

Evidence from Nerve Transplantation Studies for a Primary Schwann Cell Disorder in Charcot-Marie-Tooth (CMT) Disease

A. J. Aguayo, S. C. Perkins, G. M. Bray and I. Duncan, Montreal

Human cells transplanted into immune-suppressed mice will enshealth and myelinate mouse axons (Nature 268: 753, 1977). The present study compares myelination of mouse axons by human Schwann cells grafted from two CMT patients and controls. Control human sural nerve fascicles obtained from limb amputations were grafted between the stumps of cut sciatic nerves in 38 adult mice (C57BL/6J). Segments of biopsied sural nerves from two patients with CMT, aged 16 and 52 years, were also grafted into 19 mice. All host animals received antilymphocytic serum. Quantitative ultrastructural studies from 6 weeks to 6 months after transplantation showed that most axons regenerated across the graft and into the distal stump. In control grafts however, the density of myelinated fibers was nearly 10 times that of CMT grafts. Approximately $80 \%$ of axons with diameters greater than $1 \mu \mathrm{m}$ were myelinated in control grafts while in CMT transplants only $15 \%$ of such axons were myelinated. Proximal and distal (host) stumps were well-myelinated in both control and CMT-grafted nerves.

These experiments indicate that CMT Schwann cells fail to myelinate mouse axons as effectively as controls. The appearance of the CMT grafts resembles that seen after transplantation of myelin-deficient Schwann cells from Trembler mice into normal mouse nerves (Nature 265: 73, 1977).

\section{Dorsal Root Regeneration}

T. E. Feasby, A. H. Pullen, and T. A. Sears, London, England

Regeneration in rat-lumbo-sacral dorsal roots was studied by electron microscopy at intervals up to seventy-one days following a crush lesion. Wallerian degeneration was observed at all stages up to twenty days. By eleven days, degenerating myelin was seen in both Schwann cells and macrophages.

Four centimeters central to the crush, no regenerating fibres were seen by five days. Seven days after the lesion, regenerating unmyelinated and pro-myelin fibres were observed. Myelin compaction generally occurred after the mesaxon had made 3.5 turns about the axon and this was first noted by eleven days. By seventy-one days, $69 \%$ of fibres were myelinated compared with $36 \%$ in normals. An example of two axons beginning to myelinate within the same Schwann cell was observed at twenty days.
At seventy-one days, the mean diameter of myelinated fibres was less than $50 \%$ of normal and the diameter of unmyelinated fibres was about $70 \%$ of normal. The ratio of axon diameter to total fibre diameter (' $\mathrm{g}$ ') progressively fell from 0.76 at eleven days to a normal level of 0.62 by day seventy-one. However, ' $g$ ' was directly correlated with axon diameter at the latter date and many small axons were found with disproportionately thick myelin sheaths. The extent of dorsal root regeneration is likely limited by the inability of the fibres to make synaptic connections centrally and by the lack of a vigorous metabolic response in dorsal root ganglion cells when their central processes are sectioned.

\section{The Interaction of Nerve Growth Factor (NGF) with Embryonic Chick Sensory Ganglion Cells \\ R. J. Riopelle - Kingston and Stanford}

Receptor binding and bioassay studies of beta NGF have been carried out using intact cells of embryonic chick primary sensory ganglia. Modifications of beta NGF iodination techniques and of cell preparation have resulted in minimal non-specific binding of the labelled ligand (less than $5 \%$ of total binding).

The analysis of steady state and kinetic binding data reveals two saturable binding sites with dissociation constants of $\mathrm{KDI}=2 \times 10-11$ and KDII $=1.7 \times 10^{-9} \mathrm{M}$. Site number ratios are $1 / 11: 1 / 7-15$. That the two sites are distinct and not the result of negatively cooperative interactions within a homogeneous class of receptors has been shown directly and indirectly.

Ganglionic neuronal cells have receptor sites I and II while the nonneuronal population has only site II.

The study of the biological relevance of the two receptor sites on neurons has been approached by the use of a culture system in which NGFresponsive neurons can be maintained in vitro in the absence of nonneuronal cells.

Two observations strongly suggest that site I is responsible for the (regenerative) growth of neurites in response to beta NGF: half-maximal neurite outgrowth occurs at $7-10 \%$ occupancy of site I but at only .07\% occupancy of site II; des-arginine beta NGF has the same biological properties, and binding properties for site I, as beta NGF, but it binds to site II with a dissociation constant of KD $=10-{ }^{8} \mathrm{M}$.

The biological role of site II is under investigation.

\section{Freeze-Fracture Studies of Naked Axons in the Spinal Roots of Dystrophic Mice}

G. M. Bray, M. J. Cullen, A. J. Aguayo, and M. Rasminsky, Montreal and Bethesda

The spinal roots of dystrophic mice which contain groups of naked axons also conduct impulses abnormally. Axonal plaxma membranes have therefore been examined by thin-section and freeze-fracture electron microscopy of the L3-L5 spinal roots from 30 dystrophic mice (C57BL/6J $\mathrm{dy}^{2 \mathrm{~J}} / \mathrm{dy}^{2 \mathrm{~J}}$ or $129 \mathrm{~B} 6 \mathrm{~F}_{1}-\mathrm{dy} / \mathrm{dy}$ ).

In normal peripheral nerves there are many particles on the protoplasmic (P) face of the internodal axonal plaxma membrane but few on the external (E) face. At nodes of Ranvier, many particles are associated with the axonal E-face. In dystrophic spinal roots, aggregates of E-face particles are observed at irregular intervals along the naked axonal segments. In addition, the naked axons show particle-free membranous protrusions.

Because E-face particles at nodes of Ranvier are thought to indicate ionic channels, the presence of similar particles in non-nodal regions of naked axons in the spinal roots of dystrophic mice may relate to the ability of these naked axons to sustain continuous conduction of nervous impulses (Rasminsky, Kearney, Aguayo and Bray, Brain Res., in press).

\section{CLINICAL NEUROLOGY I (Multiple Sclerosis)}

\section{Linoleic Acid in Multiple Sclerosis: Failure to Show any Therapeutic} Benefit*

D. W. Paty, S. Read, R. K. Cousin, and K. Adlaka, London

Linoleic acid (LA) has been suggested as a treatment for Multiple Sclerosis (MS). Millar and co-workers (BMJ, 1973, 1, 765) have reported that the frequency and severity of relapses in MS was significantly reduced by an oral supplement with $\mathbf{L A}$ in a controlled trial. We set out to confirm Millar's findings in a double blind trial using sunflower seed oil (66\% LA) as the source of LA and olive oil ( $83 \%$ oleic acid - OA) as the control. The 
amount and preparation of the oil was exactly as in Millar's study. Ninetysix patients with clinically definite MS began the trial. Twenty patients withdrew for various reasons leaving 76 patients finishing the full 30 months of the trial. LA and OA blood levels were obtained at each visit. Clinical assessment was by neurological examination, assignment of the Kurtzke disability and functional scales, and by 50 timed tests of standard upper and lower extremity functions. There were 38 patients in each of the LA treated and $\mathrm{OA}$ treated groups. The initial Kurtzke disability rating, age of onset, and duration of the disease were the same in each group. The blood levels in the stable MS patients were the same as in normals. Neurological controls in hospital and acutely relapsing MS patients had lower blood levels. We regret that we were unable to show any benefit from the LA treatment on relapse rate, severity of relapses, performance on the functional studies, or on overall disability. We do think that the use of timed functional tests in serial evaluations of neurological disability is valid.

*This study was supported by the MS Society of Canada.

\section{Disseminated Lupus Erythematosis: Nervous System Involvement Including Multiple Sclerosis}

\section{J. MacFayden and B. Rozdilsky, Saskatoon}

A recent review of those fatal cases of DLE at the University Hospital in which an autopsy was performed revealed 15 patients over the past 20 years. Ten were females and 5 were males. A wide range of nervous system symptoms and signs was present prior to death. Seizures occurred in 5 patients, catastrophic intracerebral hemorrhage in 2, paraparesis in 2, progressive bilateral visual loss in one, and schizophrenia in one. With the exception of the latter, one patient with seizures and one patient with a history of amaurosis fugax, all patients with neurological symptoms or signs had nervous system pathology (8 patients). An additional 2 patients with no recorded neurological signs had brain pathology unrelated to their DLE (microabscesses and ischemic infarcts secondary to major vessel atherosclerosis). Only 5 patients had no nervous system pathology at autopsy.

Of the 8 patients with nervous system signs and pathology 2 had massive intracerebral hemorrhages likely secondary to their DLE-associated hypertension, 6 had CNS vasculitis typical of DLE and/or microinfarcts and one with no CNS vascular lesions had the typical findings of multiple sclerosis.

The history, signs and pathology of this patient with DLE and multiple sclerosis is described in some detail. The possible relationship of these two immunologically determined diseases is discussed.

\section{A Trial of Baclofen in Treating Spasticity Due to Multiple Sclerosis}

$$
\text { G. M. Sawa and D. W. Paty, London }
$$

Baclofen,* a GABA agonist, has been widely used outside North America to effectively relieve spasticity in neurological patients. The reported side effects were mild and infrequent. In this study a double-blind crossover placebo-controlled format was employed to evaluate the effectiveness of Baclofen in relieving lower-limb spasticity was eighteen Multiple Sclerosis (MS) patients. Spasticity was graded by two independent observers, utilizing a simple objective assessment of tone in the extensor muscles of the knees. Activities of daily living, which would be adversely affected by spasticity, were also evaluated. Blood pressures, body weight, liver, renal and hematological parameters were monitored. Maximum oral doses of $60 \mathrm{mg}$./day (in 3 divided doses with meals) was attained by most subjects. The active agent and placebo were given for three weeks, between which there was a seven-day washout period.

While on placebo, no subjects exhibited a detectable change in spasticity; whereas, nine of fifteen subjects, while on the active agent showed an objective improvement in spasticity ( $p$ less than .001 ). Such side effects as sedation, nausea, and increased weakness were more common than that reported in the literature but were well tolerated by most subjects. No serious toxic reactions occurred. Two patients withdrew because of side effects and one because an exacerbation of MS had occurred. It was often apparent when the active agent was being taken because the side effects were frequent and were associated with a clearly detectable relief of spasticity. Baclofen proved an effective and safe drug in treating spasticity in patients with MS. It was most beneficial in those patients who did not require spasticity for support in walking. It was also quite beneficial in patients with extensor or flexor spasms which hindered self and nursingcare.

- Lioresal (CIBA-GEIGY)

\section{The Double-Flash Test for Visual Involvement in Multiple Sclerosis: A Possible Pathophysiological Basis \\ D. M. Regan and T.J. Murray, Halifax}

Multiple sclerosis (MS) impairs a patient's ability to detect that two closely-following flashes of light are double rather than single. By using a test field of small size $\left(0.3^{\circ}\right.$ diameter), both the severity and the angular extent of double-flash field defects can be mapped. (By "severity" we mean the extra interval between the two flashes required to enable the patient to see them as double). These two measures may separately monitor increases in the degree of demyelination and increases in the number of axons affected. Although it is not specific for MS, the double-flash test can aid diagnosis by detecting subclinical visual pathology and the test seems to be more sensitive than evoked potential tests.

Visual perception is delayed in MS. Closely-adjacent points in the visual field may have very different delays (1). MacDonald (2) suggested that these irregular delays would desynchronise flicker signals from different parts of the visual field, and thus reduce critical fusion frequency: his suggestion could also explain degraded double-flash perception. Our alternative explanation was that, if the maximum firing frequency of visual axons were reduced by demyelination (as it is known to be in animal preparations) then short intervals between flashes would no longer be clearly signalled by the axons. MacDonald's explanation predicts that, as the stimulus field is made smaller (so that very few axons are involved), MS patients should more closely approach normal double-flash perception. Our explanation does not predict this. Our preliminary experimental findings are that, for most MS patients, double-flash threshold less closely approximated control values as field size was progressively reduced. This finding weighs against MacDonald's explanation in its pure form. A complication, however, is that this finding did not hold for all MS patients.

References: (1) Brain 99: 43-66, 1976

(2) Brain 97: 179-196, 1974

\section{NEUROSURGERY I (Diagnostic Aids)}

\section{Computerized Tomography (C.T.) of the Cervical Spine and Spinal Cord} C. Gonsalves, Alan Hudson, W. J. Horsey and W. S. Tucker, Toronto

This paper reports the experience gained in scanning the neck, using a first generation body scanner. The major technical difficulty results from the natural cervical lordosis. This means that not every cut passes at right angles to the spinal canal. A second problem is that the scan duration is $41 / 2$ minutes, and this may result in movement artefact. Despite these difficulties, the authors have obtained satisfactory results and this experience is reported here.

The normal C. T. anatomy of the spinal cord and subarachnoid space and the normal C. T. anatomy of the vertebral canal will be shown. The presentation includes examples of plain films and myelograms of patients who have undergone cervical C. T. scanning with the accompanying black and white and coloured $C$. T. scans.

Our conclusion is that cervical C. T. scanning is a useful adjunct in evaluation of patients suffering from spinal cord problems. Syringomyelia appears to be the pathology in which this technique is most useful. The longitudinal and transverse extent of the cavity may be assessed before and after operation. In cases of spinal stenosis, the vertebral canal images are displayed over a grid which allows direct measurement of the canal dimensions. Osteophytes are clearly depicted.

\section{Metrizamide Cisternography}

\section{Cordell E. Gross, Eugene F. Binet and Robert B. King, Iowa City}

Metrizamide (Amipaque) is a mildly hypertonic, non-ionic, water soluble contrast material recently evaluated in the U.S. and Canada as an alternative to Pantopaque myelography and cisternography. The material is instilled in the lumbar theca and in a matter of minutes diffuses and mixes with the CSF, distributing contrast throughout the ventricular and subarachnoid spaces. Fifteen to twenty minutes after installation of metrizamide at concentrations of $28 \%$ iodine, hypocycloidal tomography can demonstrate even the small recesses of the cisterns. Forty-five minutes after installation, the contrast is too faint to be useful on plain X-ray examination; however, it is still sufficiently concentrated to provide exceptional enhancement of the cisterns for CT scans. This combination of hypocycloidal tomography and CT scans with metrizamide enhancement of the cisterns has proved valuable in evaluating the parasellar and 
suprasellar spaces, as well as the cisterns of the posterior fossa. Metrizamide has the obvious advantage over air in that no special maneuvers are required to position and hold the contrast in areas of interest.

Clinical material will be presented demonstrating angiograms and metrizamide enhanced hypocycloidal and computerized axial tomograms used in evaluating anatomic features related to the empty sella, sellar tumors, and brain stem tumors.

\section{Computerized Axial Tomography of Unoperated Hematoma: Short and Long-Term Follow-Up}

John Lusins, Hiroshi Nakagawa, and Morris B. Bender, New York

The authors report a total of 12 cases $(6$ recent and 6 long-term followup) with unoperated subdural hematoma studied by Computerized Transaxial Tomography. (CAT Scanning). This method was used to demonstrate progressive resolution of the hematoma in five of the recent patients as well as enlargement of the hematoma in one of the patients who subsequently was sent to surgery. CAT Scanning was also used to demonstrate absence of subdural hematoma in long-term follow-up of unoperated patients. The possible difficulty in visualizing an isodense subdural hematoma is discussed, as is the use of additional coronal views to improve subdural detection.

\section{Choroid Plexus Tumors in Children \\ Kenneth Kaan, William D. Robertson, and Jocelyne S. Lapointe, Vancouver}

A review of the current literature of choroid plexus tumors will be made with particular reference to the incidence in infancy and childhood and a review will be made of the pathology and radiology of these lesions.

Clinical cases will be presented.

In all three cases the plain film shows evidence of raised intracranial pressure with enlargement of the neurocranium and splitting of the sutures. No abnormal intracranial calcification is noted.

Computerized tomographic scanning demonstrates a slightly increased density in the trigone that is well circumscribed and enhances uniformly with contrast medium. Hydrocephalus is present with symmetric enlargement of the lateral ventricles and enlargement of the third and fourth ventricles. The subarachnoid space is also increased in size, indicating a communicating hydrocephalus. The ventricular system is not displaced. In one case entrapment of the occiptal horn is noted.

Cerebral angiography confirms the presence of hydrocephalus with stretching of the pericallosal arteries and lateral displacement of the lenticulostriate and middle cerebral arteries. The venous phase shows low lying internal cerebral veins and subependymal veins that outline grossly dilated lateral ventricles.

The tumor receives its blood supply through the anterior and posterior choroidal branches; the anatomy of these branches may be distorted. A uniform persistent tumor stain is demonstrated and lasts well into the venous phase. In one case the tumor stain persisted for at least ten minutes after contrast injection. This is thought to be due to a breakdown of the blood brain barrier in the region of the tumor with passages of contrast into the tumor.

Both the CT scan and angiographic findings are characteristic and ventriculography is not required for the diagnosis.

\section{NEUROSURGERY II (Intracranial Vascular)}

Seventy-Nine Consecutive Cases of Ruptured and Acutely Bleeding Aneurysms

V. Kleider, Windsor

Each of these cases had been treated on an emergency basis. Cerebral angiography had been done immediately following transfer from the various local hospitals, and most of the cases had been immediately followed by surgical treatment.

The cases have been classified according to an outline suggested by Dr. Botterell.

$\begin{array}{llr}\text { Grade One } & - & 1 \text { case } \\ \text { Grade Two } & - & 23 \text { cases } \\ \text { Grade Three } & - & 13 \text { cases } \\ \text { Grade Four } & - & 20 \text { cases } \\ \text { Grade Five } & - & 22 \text { cases }\end{array}$

Only cases where an aneurysm could not be visualized by conventional cerebral angiography, and the neurologically hopeless cases were medically treated.

The mortality rate for Grade One and Grade Two was $0 \%$ for Grade Three cases $7.7 \%$, for Grade Four cases $30 \%$, and for Grade Five cases $59.1 \%$.

The overall mortality was found to be $25.5 \%$.

This paper will attempt to show justification, if not need, to operate on such patients, who are often omitted from surgery because of waiting for an optimal time, or because of the classification and expected rather high mortality rate. The results indicate that a significant number of those patients may be salvaged - patients who otherwise would probably die.

\section{Carotid-Ophthalmic Aneurysms: A Review of 53 Cases G. G. Ferguson and C. G. Drake, London}

Fifty-three patients with carotid-ophthalmic aneurysms form the basis of this review. The following distinctive features were noted:

1 . There was a large female preponderance $(85 \%)$.

2. The majority of the aneurysms were "intact" $(60 \%)$. Of these, one-half presented with major visual symptoms, while the remainder were asymptomatic.

3. One-third of the aneurysms were of "giant" size (greater than $2.5 \mathrm{~cm}$ ). Of these, the majority $18 / 19$ were symptomatic.

4. In just over one-half of the cases $(30 / 53)$ there was more than one aneurysm. This was most commonly a posterior communicating aneurysm (12 cases) on the opposite side. Bilateral ophthalmic aneurysms were present in 9 cases.

The surgical treatment of these aneurysms often presented a major technical challenge because of their size, their proximal position to the carotid artery, and the desire to adequately decompress the visual apparatus. Techniques used included carotid ligation (7), ligature and/or clipping of the neck (36), trapping with or without EC/IC bypass (3), packing (3) and exploration only (4).

The results are encouraging, however. For the entire series the outcome was excellent in $65 \%$ of the cases and good in $15 \%$; while $20 \%$ of the patients are poor or dead. Of the 16 patients with intact aneurysms presenting with visual impairment, improvement in vision occurred in 9 , while there was no change in 5 , and a worsening of vision in 2 .

\section{The Management of Carotid Cavernous Fistula}

R. Ford, P. Richardson, D. Anderson, Montreal

Five cases of carotid cavernous fistula will be reported. Two patients were immediately blinded in one eye by the trauma. Three had unilateral impaired vision due to proptosis and cranial nerve palsies.

All five cases were treated by isolation of the fistulous segment. The Fogarty balloon was inflated distal to the fistula and the proximal segment of the artery was embolized with gelfoam. Repeated intraoperative angiography was performed. The internal carotid artery was ligated in the neck over the catheter and the catheter was clipped and sutured into the sternomastoid muscle. EEG was used as a control intraoperatively.

In cases 4 and 5 an EC-IC bypass was performed before occlusion of the fistula. The bypasses were shown to be patent before the embolization.

Proptosis resolved within weeks postoperatively and the cranial nerve palsies within months. Eye findings will be reported in detail.

The ideal treatment for carotid cavernous fistula is to occlude the fistula and preserve the blood supply to the brain as Parkinson and Hosobuchi have achieved. EC-IC bypass before occlusion of the fistula protects the blood supply to the endangered hemisphere intra-operatively and in the longer term.

\section{Direct Intraoperative Embolization of Cerebral Arteriovenous Malformations}

\section{S. J. Peerless, C. G. Drake, and J. Allcock, London}

Six patients with large and otherwise inoperable cerebral arteriovenous malformations have been treated by intraoperative direct, selective injection of feeding arteries with large volumes of Gelfoam-Hypaque emboli.

Complete obliteration of the abnormal vasculature and the arteriovenous communication has been achieved in 4 cases and almost completely in 2 . One patient died post operatively of pulmonary complications. Two patients suffered a significant but fortunately transient increase in neurologic dysfunction following the procedure. The remaining patients all 
have remained well, free of their presenting headache and recurrent hemorrhage.

The details of the operative technique and the advantages over indirect, flow-directed methods of embolization will be discussed.

\section{NEUROMUSCULAR II}

\section{Midbrain Asterixis: Clinical and Electromyographic Observations} Vera Bril, James A. Sharpe, Peter Ashby, Toronto

Asterixis has been considered a pathognomonic sign of metabolic encephalopathy. Reports of unilateral asterixis after ventrolateral thalamotomy (Young et al, Trans Am Neur Assoc 101:306, 1976), and with mesencephalic dysfunction in a patient with hydrocephalus (Tarsy et al, Arch Neurol $34: 446,1977)$ suggest a discrete neuro-anatomic substrate for this intermittent lapse of postural tone. We report bilateral asterixis from midbrain infarction in a patient without any metabolic disturbance.

Complete bilateral ptosis was the presenting feature of midbrain ischemia in a 71 year old man. Complete right third nerve palsy, and vertical ophthalmoplegia confirmed discrete midbrain tegmental involvement. Leftward gaze paresis and midbrain corectopia of the left pupil signified damage to the reticular formation. Asterixis of both hands was documented by cinematographic and electromyographic analyses. Alertness was maintained, and there were no sensory or other motor deficits. Hepatic, pulmonary and renal function tests were normal. Electroencephalography showed normal alpha activity with intermittent bisynchronous theta waves. Cessation of extensor and flexor forearm muscle potentials corresponded to the lapses of hand posture. Lapses were followed by synchronous bursts of activity in flexors and extensors at 10 hertz. We postulate that asterixis results from episodic failure of postural control by the reticular formation. Our observation indicates that asterixis can be a sign of structural brainstem disease.

\section{A Familial Neuropathy Presenting with Muscle Cramps}

R. F. Nelson, T. W. Picton, V. J. Montpetit, H. Rabinovitch, Ottawa

Patients presenting with muscle cramps may be suffering from a variety of diseases but most cases are of benign etiology and only rarely is there demonstrable neuromuscular pathology. Members from three generations of one family have suffered from muscle cramps. There are no other symptoms or signs in the younger members of the family although the eldest patient had weakness and wasting of both proximal and distal muscles. The family tree suggests an autosomal dominant mode of inheritance. Nerve conduction velocities were quite slow - between $20-30 \mathrm{~m} / \mathrm{s}$. EMG examination showed evidence of chronic denervation and re-innervation. There were post-exertional fasciculations, and during cramps "doublet" motor unit patterns were noted, both findings suggestive of some increased irritability of the peripheral nerve fibres. Muscle biopsies showed severe denervation atrophy and nerve biopsies showed segmental demyelination and occasional onion-bulbs.

\section{The Limitations of the F Response to Measure Proximal Conduction Velocities}

S. K. Yates and W. F. Brown, London

The ' $F$ ' motoneurone discharge has been used to measure the motor fibre conduction velocity over proximal segments of the perpheral nerves and roots, segments not otherwise testable except by use of spinal evoked potentials. The usefulness of the ' $F$ ' discharge depends on how representative motor units in the ' $F$ ' response are of the motor units. that determine the maximum motor conduction velocity in the conventional ortho-dromic test method. By means of a variety of electrophysiological methods, it has been learned that for stimulus trains of 100 or less, only about $1 / 4$ motor fibres have an ' $F$ ' response, only $1 / 10$ having an ' $F$ ' for the short stimulus trains ( 10 or less) used by most authors to measure proximal conduction velocities.

Higher stimulus intensities recruited larger motor units into the ' $F$ ' discharge. Increments in the stimulus intensity increased the ' $F$ ' discharge frequency in some motor units, reducing it in others. Unlike the $M$ response, where there was significant relation between motor unit surface voltage and latency, there was no significant relation between the motor unit surface voltage and the corresponding ' $F$ ' latency. The observations that only a fraction of motor units have an ' $F$ ' discharge, wide range in ' $F$ ' motor unit latencies and lack of a relation between ' $F$ ' latency and MU surface voltage suggests that considerable caution should be used in the acceptance of the ' $F$ ' wave to measure proximal conduction velocities in man.
Infantile Weakness and Respiratory Failure due to Myotubular Myopathy

A. K. W. Brownell and I. R. Burgess, Calgary

A male infant weighing 3225 grams was born at term after a pregnancy characterized by excessive weight gain, hydramnios, and decreased fetal movement. From birth, the child was noted to be profoundly weak and to have inadequate respiratory effort. Muscle bulk appeared decreased, flexion contractures were noted at the hips and knees, micrognathia and a small mouth were observed, the fingers were long and tapered and the testes were undescended. Eventration of the diaphragm was noted by $x$-ray. The diagnosis of a congenital myopathy was considered but the child died before a muscle biopsy could be done. At autopsy, histologic examination of skeletal muscles revealed changes characteristic of myotubular myopathy. Although asymptomatic, the mother demonstrated mild proximal limb and neck flexor weakness, moderate elevation of creatine kinase, a myopathic electromyogram, and nonspecific myopathic changes on muscle biopsy.

\section{NEUROBIOLOGY II}

Estrogens and the Extrapyramidal Motor System

P. Bedard, P. Langelier, A. Villeneuve and B. Raphy, Quebec

Estrogens have an inhibitory action of the hypophyseal dopamine receptor, responsible for controlling prolactin secretion (Raymond, V., Labrie, F. and others, Science, in press).

We report here the effect of conjugated estrogens 0.625 to $1.25 \mathrm{~ms}$ p.o. id in six patients with Parkinson's disease and DOPA induced dyskinesias and one patient with a phenothiazine induced oral tardive dyskinesias. Estrogens improved markedly the dyskinetic phenomenon but decreased the effect of L-DOPA in 3 Parkinsonian patients.

A series of castrated female rats with a lesion of the left entopeduncular nucleus were tested several times for circling with apomorphine $0.5 \mathrm{mg}$. s.c. The same tests were repeated after administration of estradiol $1 \mu \mathrm{g} \mathrm{s.c.} \mathrm{for}$ seven days. There was a significant reduction in circling.

The present results, experimental and clinical suggest an inhibitory action of estrogens on striatal dopamine receptors. This may have useful clinical applications.

\section{Effects of Lesions along the Cholinergic Tracts of the Limbic System: An Experimental Study in the Cat \\ Andre Olivier and Richard Smith, Montreal}

Lesions were made in the septal nucleus, fornix, cingulum and longitudinal striae in the Cat and their effects studied on the cholinesterase content of the mesial hemispheric wall and the hippocampal region, as demonstrated by Koelle's thiocholine histochemical technique.

Lesions involving the septal nucleus and/or fornix cause a striking disappearance of stained fibers in the hippocampus and para-hippocampus regions with a topical arrangement. Lesions of the cingulum cause a marked proximal accumulation of the enzyme and a widespread disappearance of the enzyme along the posterior extent of the cingulate gyrus and mesial hemispheric wall including the occipital lobe, and retrosplenial gyrus. The longitudinal striae appear to terminate mainly in relationship with the dentate gyrus.

The findings of the present study give further evidence for the existence in the Cat of a large dual cholinergic system arising from the septal area and projecting to the mesial hemispheric wall via the cingulum and to the hippocampal formation via the fornix. The results are in agreement with the work of Shute and Lewis describing a similar cholinergic system in the Rat (1967) and the description by Krnjevic and Silver (1965) of cholinesterasic fibers in the limbic structures of the Cat.

\section{A Neuroanatomical Study of a Caudally Directed Pathway Issuing from the Basal Ganglia in Cat \\ Haring J. W. Nauta, Toronto}

The corpus striatum is thought to participate in the central control of movement and posture but it remains unclear by what neutral substrates it eventually comes to influence the bulbar and spinal motor neurons. One such substrate appears to involve the massive rostrally directed limb of the ansa lenticularis terminating in part in the thalamic nuclei ventralis lateralis and ventralis anterior and thus allowing a major influence on the motor cortex. Less well understood are the caudally directed pathways issuing from the basal ganglia. 
In the present experiments using the auto-radiographic tracing method it was possible first to define the course of the descending limb of the ansa lenticularis and its termination in a circumscript area of the midbrain reticular formation corresponding to the nucleus tegmenti pedunculopontinus, pars compacta (TPC). Second, it was possible to define some efferent projections of this midbrain reticular area. From the data it appears that the major descending projection of the nucleus TPC is to the medial two thirds of the pontine and medullary reticular formation - structures known to have major direct connections with the spinal cord. Only a very small number of axons issuing from the nucleus TPC extend caudally beyond that medulla into the spinal cord itself.

Details of these and other projections of the nucleus TPC will be discussed.

\section{Chromaffin Cells in Culture Resemble Adrenergic Neurons}

\section{M. Dean, G. M. Bray and B. G. Livett, Montreal}

Because peripheral adrenergic neurons and adrenal medullary chromaffin cells have a common embryological origin in the neural crest, isolated adrenal chromaffin cells provide a model system for investigating adrenergic function. The present communication describes their purification and growth characteristics in culture. Chromaffin cells were obtained by low speed centrifugation after retrograde perfusion and incubation of dissected bovine adrenal medullae with hyaluronidase and collagenase in a HEPES ascorbate buffer. Further purification was achieved by high speed centrifugation on a Percoll gradient, resulting in a complete separation of the chromaffin cells from contaminating erythrocytes and cell debris. When plated on glass or plastic surfaces under conditions that have been shown to favour sympathetic neurite outgrowth in culture, these cells attached within $3 \mathrm{~h}$ and by $8 \mathrm{~h}$ had extended processes with growth cones which eventually made contacts with other cells. The cells and processes contained high concentrations of catecholamines as demonstrated by the Faglu fluorescence histochemical method, a procedure that permits subsequent examination of the cultures by electron microscopy. The morphological features exhibited by these cells suggest that adrenal chromaffin cells may differentiate into adrenergic neurons in culture.

\section{CLINICAL NEUROLOGY II (Tremor)}

\section{The L-Dopa On-Off Effect in Parkinson's Disease: Treatment by Partial} Withdrawal and Dopamine Receptor Resensitization

L. Direnfeld, L. Spero, J. T. Marotta, and P. Seeman, Toronto

It has been suggested that patients with Parkinson's Disease partially compensate for neurone loss by developing denervation supersensitivity, and, if so, prolonged L-Dopa therapy might lead to desensitization. As a preliminary test of this hypothesis, and in order to study whether it was possible to "resensitize" a patient who had already presumably been desensitized by previous L-Dopa therapy, one patient who had become unpredictably responsive to L-Dopa was investigated.

The patient had been taking L-Dopa for eight years, and before the study exhibited severe dyskinesia-akinesia oscillation ("on-off" phenomenon), and there was no consistent response to the hourly doses of Prolopa (L-Dopa and Benserazide in a 4 to 1 ratio, manufactured by Hoffman-La Roche Ltd.). He was first lowered, over 33 days, to $20 \%$ of his original Prolopa dose. The dose was then increased until a consistent response was observed. The three main results achieved were firstly, the overall reduction by $64 \%$ in the daily requirement of L-Dopa; secondly, the conversion from a previously unpredictable response to a predictable response to each dose of L-Dopa; and, thirdly, fluctuations in movement became more typical of "end-of-dose" akinesia than the "on-off" phenomenon.

The results support the idea of dopamine receptor resensitization upon reduction of the L-Dopa dosage.

In addition, the effect of diet and the autonomic nervous system in causing post-prandial akinesia in Parkinsonian patients is discussed. Dietary amino acid content is probably much less important than a central increase in parasympathetic-cholinergic tone seen post-prandially.

\section{Pharmacotherapy for Dystonia Musculorum Deformans}

Peter Humphreys and Gordon V. Watters, Montreal

Responses to drug therapy in three boys with dystonia musculorum deformans are discussed. Two French-Canadian boys with Eldridge type IV-A torsion dystonia both responded dramatically and completely to sm. all doses of L-DOPA. A third boy with Eldridge type I-B dystonia failed to respond to both L-DOPA and haloperidol but showed a significant response to carbamazapine. All have had sustained improvement for at lease $18 \mathrm{mo}$. (range $18 \mathrm{mo}$. to 6 years); two (cases 1 and 3) deteriorated markedly when respective medications were stopped. Collated clinical experience suggests that dystonia musculorum is really a group of disorders, often hereditary, each with a distinct pathophysiologic basis. One such subgroup is responsive to dopamine agonists while others are not; some of the latter respond instead to other agents particularly to carbamazepine. Our experience suggests that patients with dystonia musculorum deformans must be considered for therapeutic trials with various agents, including LDOPA and carbamazepine, before any thought is given to stereotactic thalamotomy.

Essential Hypertension (HT) and Essential Tremor (ET) A. H. Rajput, T. W. Wilson, D. J. MacFadyen and B. Rozdilsky, Saskatoon

Essential tremor (ET) is generally regarded as a mono-symptomatic disorder. However recent studies show a high incidence of spasmodic torticollis (ST) in ET patients. Although ET and ST each are well recognized clinical entities, the pathophysiological basis is unknown in both these conditions. We have observed increased incidence of hypertension (HT) in ET patients. During a nine-year period 119 ET patients (64 males and 55 females) were evaluated by one of us (AHR or DJM); 43 were examined on at least two occasions, while 76 were evaluated only once. Blood pressure (BP) was recorded after five-minute rest in the supine position. Diagnosis of HT was made if the patient took antihypertensive drugs or a single pressure reading was higher than both systolic and diastolic criteria for the age (under 40 ) $=140 / 90,40-60=160 / 95$, over $60=165 / 100$ ). Thirty-one (26\%) of these patients had HT. This prevalence rate of HT in patients with ET is much higher than $7 \%$ found in a local mass blood pressure survey. While those ET patients who suffered from HT could not be distinguished by neurological features, the prevalence of head tremor was slightly higher (68\% vs $49 \% \mathrm{P}=\mathrm{NS}$ ) in this group. One ET patient who suffered from HT died of leukemia. At autopsy gross and histological examination of the brain and spinal cord (CNS) was normal. One possible explanation for unusually high incidence of HT in ET is increased activity of the sympathetic nervous system (SNS) in these patients. Other factors which support this hypothesis are: strong association of ET with dystonia, elevated serum dopamine beta hydroxlase (DBH) levels in dominantly-inherited dystonia, and elevated DBH in some patients with HT. While the CNS is histologically normal in ET, studies to evaluate the functional state of SNS in this condition are necessary.

\section{The Role of Peripheral Versus Central Mechanisms in Parkinsonian and Essential Tremor}

R. G. Lee and R. B. Stein, Calgary and Edmonton

The purpose of this study was to investigate the extent to which certain types of pathological tremor could be "reset" by external stimuli. It was anticipated that the results would help determine the relative contribution of central oscillators and peripheral reflex mechanisms to the generation of tremor.

Recordings were carried out from ten patients with Parkinsonian tremor and eight patients with heredofamilial or essential tremor. A torque motor was used to introduce random perturbations at the wrist during ongoing spontaneous tremor. The tremor was analysed by measuring the timing of bursts of activity in the rectified filtered EMG signal from forearm muscles. Actual times of tremor bursts following the stimulus were compared to predicted times of occurrence had the perturbation not occurred. A second technique to modify ongoing tremor was to apply oscillations to the extremity at varying frequencies close to that of the actual tremor. The resulting oscillation of a handle attached to the subject's hand was analysed to determine the extent to which the tremor persisted at its original frequency and remained independent of the applied oscillation.

The results showed clearly that essential tremor can be readily reset by external perturbations, suggesting that reflex mechanisms play a significant role in the maintenance of this type of tremor. In most of the Parkinsonian patients studied, there was only a slight tendency for the tremor to be reset, suggesting that central oscillators play a relatively greater role in Parkinsonian tremor. However, some Parkinsonian patients did show a high degree of resetting and it would appear from the data available so far that the tremor of Parkinsonism may represent a spectrum in which central and peripheral mechanisms play varying roles. 


\section{CLINICAL NEUROLOGY III (Epilepsy) \\ Significance of Isolated Infantile Convulsions as a Cause of Focal Epilepsy \\ Theodore Rasmussen, Montreal}

While it is well known that prolonged febrile convulsions in infancy can produce significant brain damage, the relative importance of isolated infantile convulsions, in general, febrile or non-febrile, as a cause of focal epilepsy and in particular, tempol lobe epilepsy, is not agreed upon. About $15 \%$ of the cases of medically refractory focal epilepsy who have undergone cortical resection at the Montreal Neurological Institute have a history of one or more isolated infantile convulsions, usually associated with fever, well before the onset of the recurring seizures. In nearly a third of these patients the infantile convulsion was associated with an identifiable febrile illness (measles, encephalitis, meningitis, etc.) which probably caused brain injury and the convulsion. In another fourth of these patients there was definite brain injury at birth and the fever triggered off a seizure tendancy which was developing presumably due to a pre-existing birth brain injury. In an additional $7 \%$ some other pre-existing cause for brain injury was present. In the remaining $42 \%$ of the patients with a history of isolated infantile convulsions there was no other obvious cause for brain injury and epileptogenicity. These patients represent $7 \%$ of the total patients in this surgical seizure series. These data suggest that isolated febrile infantile convulsions, not associated with an identifiable brain disease, are a potential cause for a small but definitive percentage of cases of focal epilepsy. Somewhat more frequently a febrile episode in childhood triggers off a maturing seizure tendency due to some pre-existing brain injury or lesions.

\section{Pain as a Symptom of Cerebral Seizures}

G. B. Young and W. T. Blume, London

Pain is an ill-recognised symptom of cortically originating seizures. We describe seven patients in whom pain constitutes an ictal symptom. These patients met all of the following criteria: (1) the patient used the term "pain" in describing his ictal symptoms, (2) pain occurred first or among a series of symptoms generally accepted as ictal; post-ictal pain syndromes were excluded, and (3) the EEG showed epileptiform and/or other focal abnormalities

Pain was the first symptom of the ictus in five cases and was preceded or followed by other ictal symptoms in the other two.

Correlations were made between the site of pain and the site of presumed seizure origin combining data obtained from clinical analysis, EEG, neuroradiology and neuropsychology.

A group of three patients had pain limited to the calvarium. They all had focally-originating seizures but from different cortical regions. In two cases the pain was ipsilateral to the presumed hemispheric origin of the seizure. Generalized pain occurred in the other patient.

A separate group of four patients had pain involving the arm or face unilaterally. Clinical and laboratory data localized the source of the ictus to the contralateral parietal-central region. One patient whose seizures began with left arm pain had an active spike focus in the "arm area" of the right Rolandic region at electrocorticography. Resection of this area abolished the ictal symptoms.

While calvarial pain likely represents an ipsilateral vascular response to a seizure in any part of the hemisphere, non-calvarial laterlised pain likely represents ictal activity in the parietal central region of the contralateral cerebral hemisphere.

\section{Clinical Experience with Valproic Acid}

B. J. Wilder, J. Bruni, L. J. Willmore and H. J. Villarreal, Gainesville, Florida

Twenty-five patients with uncontrolled absence seizures were treated with valproic acid in an acute twelve week single-blind, two week placebo lead-in study and continued into a long term study which is still in progress. The initial dose was $7.5-13.6 \mathrm{mg} / \mathrm{kg} /$ day and the maximal dose was 17.9 $62.5 \mathrm{mg} / \mathrm{kg} /$ day. Serum levels were generally $60-100 \mu \mathrm{g} / \mathrm{ml}$. The overall frequency of absence seizures was reduced an average of 84 percent; eleven patients experienced complete seizure remission. Similar reductions occurred in patients also having generalized tonic-clonic and myoclonic seizures. There was less improvement in the frequency of partial seizures. A moderate or marked reduction in total seizure frequency occurred in twenty-one of twenty-four patients (one patient was excluded because of non-compliance). Six hour telemetered electroencephalographs showed that
82 percent of the patients had a marked reduction in total number of three per second spike and wave discharges. There was good correlation between clinical and EEG responses.

Side effects included transient gastrointestinal complaints, somnolence, fatigue, a mild dose-related postural and resting tremor, mild alopecia, weight gain, mild thrombocytopenia and dose-related elevation of SGOT and SGPT in four of twenty-five patients.

Pharmacokinetic analysis indicated a serum half-life of six to eight hours in most patients. The relationship between dosage and serum concentration was fair (Corr. Coeeff. $=.349$ ).

During valproic acid therapy and concurrent administration of phenytoin and phenobarbital, significant interactions occurred. In ten of fifteen patients total serum phenytoin levels decreased while patients remained on a constant daily dose. Phenobarbital dosages had to be decreased in eleven of thirteen patients because of rising serum concentrations and the development of sedation.

\section{Serum Amino Acids in 3/sec. Spike-Wave Epilepsy}

Najma Aslam, Julius D. Metrakos, Katherine Metrakos, and Nico M. van Gelder, Montreal

The serum levels of 14 amino acids were determined in 44 probands with $3 /$ sec. spike-wave epilepsy, and in 22 non-epileptic control probands. The serum levels of aspartic acid, phenylalanine and taurine were significantly decreased, whereas glutamic acid levels were significantly increased in the epileptic probands. Ten ratios of metabolically related amino acids were also determined. The ratios (TAU/GLU), (THR/SER), (THR/GLY), and (THR/SER) (THR/GLY) were all significantly decreased in the epileptic group. When a discriminant function analysis was done on all of the variables (amino acids and ratios), 97\% (64/66) of the probands were correctly classified. Only one epileptic and one control were misclassified. When the probands were matched for age and sex, there was a $100 \%$ correct classification employing as few as 5 variables. The following conclusions appear warranted: (1) the amino acid metabolism as measured in the serum, is significantly altered in $3 / \mathrm{sec}$ spike-wave epilepsy; (2) the altered metabolism involves imbalances between metabolically related amino acids; and (3) discrimination into epileptic and control improves when probands are matched for age and sex, strongly suggesting that at least some serum amino acid levels are age and sex dependent.

\section{K. G. MCKENZIE MEMORIAL AW ARD}

Recipient: Dr. Richard Branan, Montreal

\section{Velocity Interval Analysis by Computer (VIAC): A New Technique for Studying Rapid Hemodynamic Changes Applied to the Cerebral Circulation}

Richard C. Branan, Carl Dila, Ronald S. Poulson, Charles P. Hodge, and William Feindel, Montreal

Due to the inherent complexity of the cerebral circulation and to the limitations of current methodology, investigations of the vasculature has concentrated on conditions producing a change in cerebral blood flow. However, little is known about the mechanism responsible for the regulation of the cerebral circulation. This is in part due to the poor spatial and temporal resolution obtained with the current technology. No known method can illustrate the rapid, transient changes with sufficient temporal and spatial resolution needed to elucidate the complex reactions of the system. In order to overcome these deficiencies, a new approach is presented which provides a vast improvement in both spatial and temporal resolution and in addition, makes available a large number of data points for statistical analysis in a form usable by a digital computer.

\section{FRANCIS McNAUGHTON PRIZE}

\section{Recipient: Dr. Serge Gauthier, Montreal}

\section{Neural Regulation of Adrenal Tyrosine Hydroxylase}

S. G. Gauthier, J. P. Gagner and T. L. Sourkes, Montreal

The importance of adrenal tyrosine hydroxylase (ATH) as a component of the regulation of normal blood pressure and possibly as an inducer of sustained hypertension has been recognized. In order to understand the regulation of ATH through the sympatho-adrenal preganglionic neurons (SAPN), a model of spinal cord transection at T3 level in 350 grams Sprague-Dawley rats has been established, by which the cord segments 
(T8-TII) projecting to the adrenal medulla are isolated from suprasegmental influences. From 3 days post-section till at least 10 days, ATH activity is $76 \%$ of sham-operated animals. This plateau of activity probably reflects the base-line output of decentralized SAPN.

To study the mode of regulation of SAPN, various centrally acting direct agonists have been injected subcutaneously during the 3 days following cord transection. At 3 days, no modification of ATH activity from spinalized base-line was found with Piribedil, a dopamine agonist. An increase was found with Clonidine, a noradrenergic agonist, and $O x-$ otremorine, a muscarinic agonist. A decrease was found with Quipazine, a serotonergic agonist. These modifications of ATH actifity suggest the presence of excitatory noradrenergic and muscarinic receptors and inhibitory serotonergic receptors in the isolated cord, possibly directly on the SAPN, as shown by histochemical and neurophysiologic studies. Dopamine receptors seem to be absent.

This model is valuable for study of the interaction of multiple neurotransmitter systems by use of a single biochemical endpoint.

\section{HERBERT JASPER PRIZE}

\section{Cortical and Cervical Somatosensory Responses in Suspected Multiple} Sclerosis

Kenneth Nudleman, Montreal

Various physiological tests have proven valuable in confirming clinically definite multiple sclerosis (MS). In suspected MS the visual evoked response gives abnormalities in about $60 \%$ of cases (Asselman et al, Brain 98: 261, 1975). This study describes the combined results of the cortical (SEPA) and cervical (SEPC) somatosensory responses in 20 MS suspects classified as early latent or probable (11 cases) and progressive possible or spinal $(9$ cases.)

SEPA and SEPC were evoked by unilateral median nerve stimulation, 128 responses being averaged. The latency of both responses was measured from the first negative (upward) deflection. From 25 aged matched controls the normal mean latency of SEPA was established to be $15.6 \pm 1.1 \mathrm{~ms}$. and of SEPC to be $10.8 \pm 1.1 \mathrm{~ms}$. Individual values were related to arm length giving regression lines with correlation coefficients of 0.81 for SEPA and 0.87 for SEPC. Eighty-two percent of the early probable group had a prolonged latency of SEPA, and in 55\% SEPC was absent or delayed. The corresponding values for the progressive possible group were $75 \%$ and $38 \%$ respectively.

$\mathrm{M}$ and $\mathrm{F}$-waves were used to calculate the transit time from wrist to $\mathrm{C}_{7}$ (TA) and the transit time from $C_{7}$ to cortex (TB), given by SEPA - TA TB was prolonged (greater than $6.9 \mathrm{~ms}$ ) in $45 \%$ of the early probable group and $38 \%$ of the progressive possible group.

Seventy percent of all the suspects had more than one abnormality. It is concluded that cortical and cervical somatosensory responses studied in combination give an incidence of abnormalities which matches that found using the visual evoked response in suspected multiple sclerosis.

\section{NEUROSURGERY III (Spinal I) Practice Profile of Neurosurgeons in Ontario}

Ross Fleming, Toronto

This paper presents a profile of the practice of neurosurgery in Ontario, the frequency with which various neurosurgical procedures have been performed in the last five years, and trends in the incidence of some of these procedures.

A unique by-product of the provincial health schemes in Canada is the availability of accurate utilization data, thanks to the fee-for-service system within a universal health care plan. The plan is billed for and records each consultation, hospital visit, surgical procedure and other services rendered. In other countries with universal health care plans, the doctors are salaried and there is no record of the individual services rendered. In countries without universal plans, the fee-for-service system and partial insurance programs prevail; however as no central agency is billed for all services, there is no central compilation of the services rendered. Thus our provincial plans are unique in their ability to provide accurate and comprehensive utilization data.

The numbers of each neurosurgical procedure billed to the provincial plan for five consecutive years have been determined, and thus the incidence of each procedure per 10,000 population per year, and the numbers of procedures done by each neurosurgeon is known. For example. there were about 9 craniotomies for tumor, and 3 craniotomies for aneurysm per 100,000 population per year. Lumbar discotomies are being done more and more by orthopedic surgeons (only $23 \%$ of the total were done by neurosurgeons in 1977), whereas anterior cervical discotomies are mostly done by neurosurgeons ( $63 \%$ of the total). Very few neurosurgeons perform diagnostic neuroradiologic procedures now.

Information of this type has not hitherto been available. It provides an interesting background against which individual neurosurgeons may compare their own activities, and a solid base for manpower planning.

\section{Prospective Study of 100 Lumbo-Sacral Discectomies Bryce Weir, Edmonton}

In August 1975 a prospective study was commenced and 100 consecutive patients were operated upon by April, 1977. Patients having previous low back surgery, multilevel laminectomies or simple dorsolateral decompression without discectomy were excluded. Where applicable, averages are given as mean \pm 1 SEM.

75 patients were male and 25 female. 72 were non-compensation cases, 28 were Workers Compensation Board responsibilities. 100 had leg pain commencing $16 \pm 3$ months prior to surgery, 93 had back pain commencing $62 \pm 7$ months. 78 were off work for $48 \pm 5$ days immediately preoperatively. 38 had previous hospitalization for low back and leg pain, 72 were taking analgesics preoperatively, 44 had previous chiropractic and 42 had previous physiotherapy. 90 had pain going into the foot, 87 had a positive cough effect, 84 had an ipsilateral decrease in straight-leg raising, 72 had parasthesia in the leg, 64 could forward bend as far as the knee only, 61 had weakness, 40 had a unilateral decrease in ankle-jerk and 18 had a contra-lateral decrease in straight-leg raising.

On preoperative myelograms, $8 \%$ had a complete block, $5 \%$ large bilateral defects, $47 \%$ large unilateral defects, $34 \%$ root cut-off or distortion, $3 \%$ hourglass deformity, $1 \%$ central defect and 1 was normal. At surgery 38 had protrusions, 24 had subligamentous extrusions, 25 had sequestration through a rupture in the posterior ligament and 11 had migration of a free fragment away from the point where it had ruptured through the posterior ligament.

There was no operative mortality or morbidity. No patient required operative transfusion and there were no infections. Surgical time was $35 \pm$ 3 minutes. The duration of postoperative hospital stay was $5 \pm .3$ days. Return to work was $40 \pm 5$ days.

In the one year follow-up $95 \%$ of patients reported they were glad they had the operation, 4 had back pain which was unchanged and one had back pain which was worse. One patient reported leg pain the same, and three said it was worse.

\section{Results of Chemonucleolysis in Disc Patients}

H. Schutz, J. F. R. Fleming, G. Vanderlinden and M. Hogan, Toronto

The long-term results of chemonucleolysis were analyzed in 71 patients. The mean age was 45 . Leg pain was the principal complaint in 56 of 71 patients and back and leg pain in the remaining 15 patients. None had back pain alone. The average duration of the present attack was five months and the average number of attacks was two per patient. Evidence of nerve root irritation, weakness, numbness and reflex abnormality was present to a varying extent in every patient. An appropriate myelographic defect was present in 53 of 71 patients. Other evidence of disc herniation (venogram, discogram) was present in 14 . The myelogram was normal in 4 . None had previous surgery. The L3-4 level was injected three times, the L4-5 level 46 times and the L5-S1 level 26 times. There were no complications. Followup was complete for 69 of 71 patients. Minimum follow-up was 9 months and maximum 4 years with a mean of 2 years. The results were evaluated by an independent investigator (M.H.).

Successful outcome (return to work or return to usual activity) was achieved in $65 \%$ of patients ( 46 out of 71 ). The outcome was unsuccessful in the remaining $35 \%$ of patients ( 26 out of 71 ). 15 patients required subsequent surgery (lateral recess syndrome, sequestrated disc) and 12 of these had an eventual successful outcome. Reasons for failure in the rest were multiple (neurosis, litigation, compensation, unknown).

An attempt was made to compare these results with standard laminectomy-discotomy for uncomplicated disc herniation with appropriate symptoms and signs in 100 consecutive patients. Patients with spinal stenosis, previous laminectomy or chemonucleolysis were excluded. The criteria for surgery were similar in all respects to the Chymopapain 
treated group. Successful outcome was achieved in $85 \%$ of patients.

In view of the present controversy, the authors wish to point out that chemonucleolysis can bring about an immediate and dramatic relief of sciatica in a certain group of patients but that surgery is more consistently and predictably effective.

\section{A rticular Facet Rhizotomy by Percutaneous Electrocoagulation}

R. G. Vanderlinden and D. Salter, Toronto

The posterior primary rami of the spinal nerves provide afferent innervation to the articular joints by a branch called the nerve of Luschka. This nerve arises near the dorsal root ganglion and runs caudally around the base of the superior facet before entering the joint capsule on its inferior surface. It has been postulated that trauma, degeneration, or inflammation of these joints may give rise to certain cases of low back pain. In 1972 , Shealy reported the technique of percutaneous electrocoagulation of the nerves of Luschka and claimed excellent pain relief in $75 \%$ of cases treated.

This paper reports a series of 30 patients with chronic lumbar or cervical pain who have undergone articular facet rhizotomy by percutaneous electrocoagulation and followed an average 15 months. A pain profile, designed to measure daily duration and severity of pain, as well as its effect on activity, personality, and drug use, was prepared on each patient. These measurements were used to evaluate the patients post-operatively and yielded the following results: Excellent (33\%), Good (24\%), and Failures $(43 \%)$.

The diagnosis in one third of patients was primary facet joint disease; the clincial, radiological and investigative criteria for this diagnosis will be presented. The technique of the procedure is relatively simple and resulted in no serious complications.

These data suggest that articular facet rhizotomy plays a definite role in the management of chronic low back pain without neurological deficit.

\section{NEUROSURGERY IV (Tumors)}

\section{The Surgical Treatment of Prolactinomas}

Giles Bertrand and George Tolis, Montreal

Thirty-six (36) female patients presenting with galactorrhea, amenorrhea and/or infertility were operated on for prolactin-secreting pituitary tumors, between April 1973 and January 1978. The surgery was carried out through a sub-labial, rhinoseptal, transphenoidal microsurgical approach.

Tumor was identified in thirty-five (35) cases and was totally removed as judged by peroperative inspection with the operating microscope and postoperative prolactin levels in 27 of the 35 . In one case where excision had been inadequate, a second operation, ten months later, resulted in complete removal of the residual tumor, bringing the percentage of adequate removal to $80 \%$ of all tumors irrespective of size or degree of invasiveness.

Although many complete adenomectomies were achieved with the larger, grade II and III tumors (10 out of $13,77 \%)$, this goal was reached more commonly in the grade 1 microadenomas. (19 of $22,86 \%)$, (90\% in cases not operated on previously).

One of two patients who also presented evidence of hypopituitarism prior to surgery improved after adenomectomy, the other remained the same. Only one patient developed hypopituitarism as a result of surgery, her tumor seemed diffuse and the contents of the sella were completely removed.

Nine patients $(25 \%)$ presented transient, self-limiting diabetes insipidus for 24 to 72 hours following surgery and one, with a previous history of polyuria and polydipsia still has diabetes insipidus requiring treatment with Pitressin.

Cerebral-spinal fluid leakage occurred during surgery in nine (9) patients and was controlled with muscle, fascia and lyophilized dura grafts. There was no leakage post-operatively except in one case where it stopped spontaneously after three days. There has been no meningitis and no evidence of sinus infection.

Perforations of the nasal septum occurred in two of the patients and two others had urinary tract infections, resulting from catheterization.

All patients who still had their teeth complained of numbness of the upper incisors, lasting many weeks.

Microsurgical removal of prolactin secreting microadenomas causing the syndrome of amenorrhea - galactorrhea and infertility in females appears to be the ideal method to re-establish normal pituitary function quickly and with minimal morbidity and risk of complication.
Subtemporal Approach to Tentorial Incisura and Interpeduncular Fossa N. F. Kassell, C. G. Drake, and S. J. Peerless, lowa City and London The development of the operating microscope, microsurgical instrumentation, and effective means for decreasing intracranial bulk provide safe access to structures at the base of the brain previously approached with difficulty. As these techniques have evolved, new surgical approaches have become practical which facilitate irradication of vascular and neoplastic lesions and minimize the risk of damage to vital structures. Access to the lateral aspect of the tentorial incisura and interpeduncular fossa including cranial nerves 3,4 and 5 and the carotid, posterior communicating, basilar. posterior cerebral, superior cerebellar artery and aneurysms thereof may be made anteriorly through the root of the sylvian fissure, laterally beneath the temporal lobe, or with a combined approach.

This paper describes a subtemporal microsurgical approach to the region of the tentorial incisura and interpeduncular fossa for the management of aneurysms and tumors. There were no epidural, subdural or intracerebral hematomas, brain contusions or lacerations, or infections in over 50 cases to date. Advantages and disadvantages will be compared with other approaches in terms of visualizations of structures, facility of instrumentation, degree of brain retraction, and damage to surrounding structures.

\section{The Effect of Phenobarbital on the Tumoricidal Activity of CCNU in a Murine Brain Tumor Model \\ P. J. Muller and C. H. Tator, Calgary and Toronto}

Others have found that the nitrosoureas are substrates for hepatic microsomal enzymes. We have previously shown in a series of experiments that four daily doses of phenobarbital (PB) administered prior to CCNU to normal and brain tumor bearing $\mathrm{C} 57 \mathrm{~B} 1 / 6 \mathrm{~J}$ mice significantly reduces the toxicity of high doses of CCNU. We concluded that hepatic microsomal enzyme induction by PB had a profound effect on the activity of CCNU. In the present series of experiments we have assessed the effect of PB pretreatment on the tumoricidal activity of standard non-toxic doses of CCNU in i.c. and s.c. tumor bearing mice.

CCNU at $15 \mathrm{mg} / \mathrm{kg}$ administered i.p. on day 5 after i.c. tumor implantation resulted in a \% increase length of survival (ILS) of 67 with a long term survival (LTS) of $2 / 25$. With the addition of 4 daily doses of $P B(75 \mathrm{mg} / \mathrm{kg}$ $x$ 4) the \% ILS was reduced to 22 . With CCNU at $30 \mathrm{mg} / \mathrm{kg}$ i.p. (the optimal therapeutic dose in this model) the \% ILS was greater than 300 and the LTS was $21 / 25$. With the addition of PB pretreatment the \% ILS was reduced to 85 with $1 / 25$ LTS. These results are significant at $p$ less than 01 .

By delaying CCNU treatment until the 10th day after i.c. tumor implantation in order to allow a greater tumor burden the PB pretreatment eliminated the survival value of 15 and $30 \mathrm{mg} / \mathrm{kg}$ CCNU. ( $\mathrm{p}$ less than .01).

The effect of PB on the tumoricidal property of CCNU was also tested in a s.c. model. In the control group, 36 days after s.c. tumor implantation, the mean tumor volume was $1841 \pm 349$ (S.E.) $\mathrm{mm}^{3}$. The administration of CCNU $30 \mathrm{mg} / \mathrm{kg}$ i.p. on day 16 greatly retarded tumor growth; with this dose the mean tumor volume on day 36 was $170 \pm 63$ (S.E.) $\mathrm{mm}^{3}$. The addition of PB pretreatment significantly (p less than .005) reduced the ability of CCNU to retard tumor growth; at 36 days the mean tumor volume of the PB-CCNU $30 \mathrm{mg} / \mathrm{kg}$ combination group was $541 \pm 91$ (S.E.) $\mathrm{mm}^{3}$.

Several hundred compounds are known to stimulate microsomal enzymes. Failures in nitrosourea chemotherapy may in part be due to unrecognized hepatic microsomal induction and subsequent unsuspected heterergic drug antagonism occurring as the consequence of altered nitrosourea metabolism.

\section{The Effect of Dexamethasone on the Plasminogen Activator Activity of Human Brain Tumors In Vitro \\ W. S. Tucker, D. Hunter, J. M. Bilbao, L. M. Fink and W. M. Kirsch, Toronto and Denver}

An increase in plasminogen activator (PA) enzyme production has been reported in a variety of transformed mammalian and avian cells in vitro. Tucker et al. have described the production of PA enzymes by human brain tumor cells in vitro (Cancer Research, 38: 297-302, 1978). Dexamethasone frequently produces symptomatic relief in patients with intracranial tumors, by reduction of perineoplastic oedma, or a direct antineoplastic effect, or both. It is possible that these effects could be mediated by a suppression of tumor PA activity. 
Human brain tumors were explanted and grown in cell culture. Electron micrographs in each case were highly suggestive that the cultured cells were neoplastic. Lysates were prepared from the cultured cells, with a total protein concentration of $1.0 \mathrm{mg} / \mathrm{ml}$. Similar lysates were prepared from cells which had been incubated with 10-9 M. dexamethasone for 24 hours. Plasminogen activator activity was determined by measuring the lytic zones produced by the lysates in fibrin plates containing plasminogen.

In a variety of primary and secondary brain tumors in vitro, dexamethasone caused complete inhibition of detectable PA activity. This phenomenon may be the basis for the beneficial effect of dexamethasone in human brain tumors in vivo.

\section{ELECTROPHYSIOLOGY I}

Spontaneous and Evoked Cerebral Electrical Activity and Localization of Language Function: A Study of Children with the Minimal Cerebral Dysfunction Syndrome

M. D. Low, L. J. Rogers, S. J. Purves and H. G. Dunn, Vancouver

During a long-term prospective study of low birth weight children, some of the subject population have been diagnosed as having the Minimal Cerebral Dysfunction Syndrome. Diagnostic criteria include birth history, neurological status, EEG findings and psychological assessment. The exact nature of the functional defect in the MCD syndrome is unknown. In light of a few suggestive reports of abnormal electrophysiological findings in such children, we have studied $20 \mathrm{MCD}$ subjects (age 14 years) using a comprehensive test set which includes a number of electrophysiological parameters during reading, preparation for speech and tasks based on the Street test and the Similarities test (shown to be right- and left-hemisphere dependent tasks, respectively).

Results of these assessments have been compared to data from a similar study of 37 normal, age matched controls. Using multi-variate statistics including discriminate analysis, the two subject groups could be distinguished with over $80 \%$ accuracy on the basis of some of the electrophysiological measures.

The most significant discriminating variables were evoked potentials to visually-presented word stimuli and the speech $C N V$, with the MCD children showing more marked left-right hemisphere asymmetries in the posterior-frontal region than normals, and with distinct intra-hemispheric differences in distribution of the potentials evoked by words. The EEG data, including coherence spectra and power spectra showed similar but less marked differences between groups.

These findings suggest that the MCD syndrome is characterized by a greater than normal "localization", or less than normal interaction both between and within hemispheres during tasks which normally require significant brain region interaction.

\section{The Pattern VEP in the Early Stages of the Diagnosis of MS: A Prospective Study \\ S. J. Purves and M. D. Low, Vancouver}

There are many reports of the diagnostic usefulness of the pattern VEP in demyelinating disease. However, most studies to date have included patients in whom the diagnosis of M.S. was more or less firmly established. This report describes the findings in all adult patients $(n=90)$ referred over a one year period for pattern VEPs, in whom the diagnosis of M.S. was suspected but not yet determined definitely. Visual acuity tested in the laboratory was $20 / 40$ or better for all patients. The median age was 36 , with $60 \%$ of the patients in their 3 rd or 4 th decades.

The pattern reversal stimulus was either a reversing black and white checkerboard, or a grid of light emitting diodes, or both. A latency value for the first major positive peak of more than $125 \mathrm{msec}$. for the checkerboard or $112 \mathrm{msec}$. for the grid stimulus, or a consistent interocular delay of greater than $8 \mathrm{msec}$. for either method, was considered abnormal.

One-third (30) of these 90 patients had abnormal VEPs. Follow-up reports indicate that at least 10 of these 90 proved to have another diagnosis (not demyelinating disease), and all of these had had normal VEPs. At the time of referral only 5 had signs and symptoms (but not necessarily in the visual system) that suggested a definite diagnosis of M.S., and all of these had prolonged VEP latencies. An additional 15 had signs and symptoms (transient or progressive) confined to the spinal cord, and of this group 3 had abnormal VEPs. Nine patients had a history suggesting a previous episode of optic neuritis (although more had questionable pallor of the optic discs) but only 5 of these had abnormal VEPs.
This prospective study of pattern VEPs in patients with suspected M.S. has shown that this test can provide an objective measure of subclinical in. volvement of the optic nerves in some patients and thus be useful in establishing the multifocal nature of the disease process at an early stage.

\section{Effect of Body Temperature on Visual Evoked Potentials and Visual Perception \\ T. J. Murray, D. Regan and R. Silver, Halifax}

It is a common clinical observation that an increase in body temperature will often worsen the signs and symptoms in patients with multiple sclerosis. Since the alterations in nerve fibre conduction seen with raising and lowering body temperature may be comparable to the conduction changes seen with demyelination and remyelination, it would be useful to assess what tests may be of value in documenting such changes. We report here a study of the effects of heating and cooling on measurements of medium frequency flicker evoked potentials, perceptual delay, double-flash resolution and modulation transfer function, i.e. grating visual acuity. We wish to determine whether such tests provide a means of monitoring the progress of demyelination and possibly also of monitoring the effects of experimental therapies.

Seven multiple sclerosis patients were cooled and four were heated. Although all had delayed evoked potentials, the evoked potential measurements changed in only five out of eleven experiments. Control limits were set by cooling eight and heating four control subjects.

One patient gave anomalous results in that although heating increased perceptual delay, degraded visual acuity, and depressed the sine wave grating modulation transfer function, double-flash resolution was improved. An explanation is proposed in terms of the pattern of axonal demyelination.

We found that, although the medium frequency flicker evoked potential test provides a reliable indication of visual pathology, evoked potential delay seems to be less affected by changes in body temperature than is either double-flash perception or perceptual delay. We conclude that the medium frequency flicker evoked potential test seems to be a less sensitive and possibly less reliable means of monitoring the progress of demyelination in multiple sclerosis patients than is double-flash campimetry or perceptual delay campimetry. However, the objective nature of the evoked potential test would be an advantage in some situations.

\section{The la EPSP in Man}

Peter Ashby and Duane Zilm, Toronto

It is possible to derive some of the characteristics of the post-synaptic potentials produced in a human mononeurone by an afferent volley from the profile of the post-stimulus time histogram (PSTH) of that neurone when it is firing rhythmically. The relationship between the shape of the post-synaptic potential and the profile of the PSTH is complex (for example, the profile of the PSTH represents roughly the first derivative of the shape of the post-synaptic potential). For this reason the interpretation of the PSTH is made much easier when certain general principals can be established and specific examples tested by computer simulation.

We have used this method to derive the characteristics of the la EPSP in tibialis anterior motoneurones in man. The rise time of the la EPSP is estimated to be less than $4.7 \mathrm{msec}$. The amplitude of the la EPSP (estimated by delivering a group 1 volley at increasing intervals after a spontaneously occurring discharge until the EPSP brings the membrane potential to threshold) is approximately $5 \mathrm{mV}$. The duration of the falling phase of the la EPSP (determined by delivering double group $l$ volleys in such a way that the second would fail to bring the membrane potential to threshold without temporal simulation from the first) suggest that the la EPSP has largely decayed $10 \mathrm{msec}$ after its onset.

\section{NEUROTOXICOLOGY}

Retrolaminar Optic Nerve Demyelination in Methanol Intoxication James A. Sharpe, N. Barry Rescastle, and Juan M. Bilbao, Toronto

The pathogenesis of visual loss after methyl alcohol intoxication has been a subject of controversy during the past century. Ensuing optic atrophy has been attributed to ascending degeneration after primary involvement of retinal ganglion cells. Recent experimental observations in the monkey indicate sparing of retinal ganglion cells and primary 
involvement of oligodendroglia in the retrolaminar optic nerve with axoplasmic stasis at the nerve head (Baumbach et al, Arch Aphthalmol 95: 1859 , 1977). We describe early histopathologic effects of methanol poisoning on the human optic nerve.

Five optic nerves from three cases of methanol intoxication were examined. The patients died in cardiorespiratory failure thirty hours, two days and eighteen days respectively after presentation with acute bilateral visual loss. The retrolaminary nerve segments showed circumscribed demyelination or loss of myelin staining with relative preservation of axons. Retinal ganglion cells were spared. Electromicroscopy in the case with shortest survival revealed periaxonal intramyelinic spaces behind the lamina cribosa. In acute retrobulbar demyelination, compression of axons by swelling of myelin sheaths may disrupt orthograde axoplasmic flow from intact retinal ganglion cells and cause optic disc swelling. We attribute subsequent optic atrophy to descending degeneration of axons from the involved retrolaminar nerve segment. In one case, areas of myelin destruction were also present in cerebral white matter. Hypoxic, ischemic or histoxic damage to oligodendroglia may be responsible for this myelinoclastic effect of methanol metabolism. Retrolaminar demyelinating optic neuropathy is an early morphological correlate of visual loss in methanol intoxication.

\section{Permanent Motor Dysfunction Following Methanol Poisoning}

D. R. McLean, B. W. Mielke and H. Jacobs, Edmonton

While visual loss with optic atrophy is the well recognized complication of methanol poisoning, permanent motor dysfunction is extremely rare.

We report two survivors of severe methanol poisoning, who apart from blindness, developed a Parkinsonian-like extra-pyramidal syndrome characterized by poor voice volume, masked facies, mild tremor, rigidity and bradykinesia. Hyper-reflexia and bilateral Babinski responses and mild dementia were present also.

CAT scan in both patients demonstrated symmetrical low density lesion in the fronto-central white matter and putamen bilaterally.

EMG showed extensive denervation, mainly involving the legs, but normal motor conduction velocities.

Autopsy in one patient who died from suicide 11 months following poisoning revealed cystic resorption of the putamen and the peripheral white matter in addition to widespread neuronal damage throughout cerebrum, cerebellum, brainstem and spinal cord.

L-Dopa adminstered to the more severely affected patient had no effect on the Parkinsonian features.

More sophisticated cardiopulmonary support equipment, hemodialysis and $a$ better understanding of balance allows recovery in patients who heretofore died from methanol poisoning. However, these patients suffer more widespread neurological damage than previously appreciated as exemplified by the unique features herein described.

\section{Bismuth Encephalopathy}

H. Jenkins, J. Crowley, I. Bowmer and N. A. Russell, St. John's

We have recently encountered three cases of this unusual syndrome. Each occurred because of the absorption of Bismuth into the C.N.S. from Bismuth Paste used to pack wound infections. These three patients developed deep wound infections after lumbar spine surgery. The usual surgical principles of incision and drainage of the wounds were adhered to. The open wounds were then packed with cotton gauze impregnated with Bismuth Iodoform Petroleum Paste (B.I.P.). This substance has been used to locally treat surgical infections for over 50 years. After several days all three patients developed a toxic encephalopathy. The clinical feature were identical. These consisted of confusion with hallucinations, unsteadiness when sitting or standing, tremulousness, dysarthria, and myoclonic jerks. Electroencephalograms were abnormal. Bismuth levels were demonstrable in the cerebrospinal fluid and in the plasma. In each patient the syndrome reversed itself completely with cessation of the use of B.I.P. packs.

These patients exhibited the characteristic features of Bismuth Encephalopathy. This condition has previously been reported to occur as a result of the therapeutic ingestion of Bismuth Salts. We are not aware of any reports of other cases resulting from the absorption of this heavy metal from a site other than the G.I. Tract. We postulate that the close proximity of the impregnated packs to the lumbar theca resulted in the relatively rapid absorption of the Bismuth into the C.N.S.

\section{Diphenylhydantoin and Pulmonary Function}

R. J. Smith, S. F. P. Man and D. R. McLean, Edmonton

Diphenylhydantoin (DPH) has been in common use since 1938. Its side effects are protean, and can affect almost every system. Controversy exists in the literature regarding the effects of DPH on the pulmonary system.

This study compares pulmonary function in two groups matched for age and sex, the study group comprising patients who have been of DPH alone for more than 2 years, and the control group comprising patients who are on other non-related anticonvulsants.

All patients answered a standardised questionaire on respiratory symp. toms as approved by the MRC Committee on Chronic Bronchitis, had a physical examination, chest $x$-ray, electrocardiogram, full blood profile, serum $B_{12}$ and folate levels, anticonvulsant drug levels, alpha, antitrypsin. anti DNA antibodies, $\mathrm{C}_{3}$ and $\mathrm{C}_{4}$ levels. Extensive pulmonary function testing was performed on all patients.

Of eleven patients in the DPH group, 2 had abnormal airway function, 2 had abnormal gas exchange, (DLco, $\mathrm{AaDo}_{2}$ ) and 3 had other minor abnormalities of pulmonary function. Of 9 patients in the control group, 1 had an abnormality of gas exchange.

The magnitude of these abnormalities, by Test of proportion, was of no statistical significance.

It is concluded that long-term DPH in therapeutic doses has no significant effect on pulmonary function.

\section{CLINICAL NEUROLOGY IV}

A Syndrome of Hemiplegic Migraine Associated with Hereditary Haemorrhagic Telangiectasia in Two Unrelated Families

Benjamin Zifkin, Frederick Andermann, Gordon V. Watters, Eva Andermann and S. Seshia, Montreal and Winnipeg

Four members of two unrelated families affected with hereditary haemorrhagic telangiectsasia (HHT) had attacks of hemiplegic migraine. In three, the hemiplegia was transient and resolved spontaneously. Lumbar punctures, cerebral arteriography and CT scans gave no evidence for subarachnoid haemorrhage, vascular malformation of intracranial mass. One patient had pulmonary arteriovenous malformations.

There was a history of classical and common migraine in other first degree relatives in both families. In a third family, migraine was also associated with HHT. In all three families, there was an increased incidence of seizures among patients with telangiectasiae.

Transient and recurrent neurological symptoms as well as acute neurological catastrophies have previously been documented in patients with HHT. The former have usually been ascribed to "hypoxia", polycythemia or air embolism; the latter to ruptured intracranial arteriovenous malformations and brain abscess.

Hemiplegic migraine has not been previously associated with a diagnosis of HHT. The occurence of both these dominantly inherited disorders in association, in two families, suggests more than a chance finding. A factor of abnormal vascular permeability rather than frank haemorrhage may lead to the attacks of hemiplegic migraine with their classical march. Recognition of this syndrome does not exclude the possibility of intracranial haemorrhage in patients with HHT, but provides an alternative diagnosis in patients with hemiparesis and severe headache.

We believe that both the neurological and haemorrhagic manifestations are due to a single autosomal dominant gene with pleiotropic manifestations. However, the possibility of two independent autosomall dominant genes cannot be excluded.

\section{Bell's Palsy - A Controlled Study with Corticosteroids}

John G. Humphrey, Toronto

278 patients with an acute non-traumatic facial palsy are reported. A control group of 71 patients was initially studied and subsequently 207 patients were selected into groups receiving corticosteroid treatment or no treatment. All had repeated clinical assessments, electromyograms and nerve conduction studies within 7 days from onset of facial weakness. The final outcome on follow-up of the control group showed A (complete recovery within 12 weeks) $4158 \%$, B (complete recovery after 3 months) $13-18 \%, C$ (incomplete recovery after 12 months) $10-14 \%$ and D (poor recovery) $7-10 \%$.

Selected patients were then divided into 2 groups within 5 days from onset of facial weakness utilizing the criteria from the control patients. 74 were felt to have a favourable prognosis $(\mathrm{A})$ and did not receive steroids. 
133 were considered to be potential B, C, or D results and received 500 units of ACTH or $500 \mathrm{mg}$. prednisone in 7 days.

Results on follow up of 74 untreated patients were A 68, B 3 and C 2 . The 133 who received ACTH or prednisone were A $102(77 \%)$, B 15 (11\%), C $13(10 \%)$ and D $3(2 \%) .15$ further patients with facial palsy and associated Herpes Zoster have received a similar corticosteroid regimen. Their results on follow-up are A 8, B 3, C 3 and D 1 indicating that steroids are less effective than in those with an idiopathic Bell's Palsy.

Prednisone was more effective than ACTH in preventing partial or complete denervation in selected poor risk Bell's palsies. It has markedly increased the incidence of early complete recovery. However it is unnecessary in many patients identified early in the course of their facial palsy.

\section{Gait Disturbance in Chronic Subdural Hematom}

\section{R. S. McLachlan, C. F. Bolton and H. J. M. Barnett, London}

Gait disturbance in chronic subdural hematoma is not well documented in the literature or current textbooks. In 1925, Putnam and Cushing described two out of their eleven cases as presenting with gait disturbance, one of whom was initially diagnosed as a cerebellar tumor. McKenzie in 1932 comments on three of his nine cases who were felt to have cerebellar lesions until ventriculography.

We undertook a retrospective study in fifty consecutive cases of chronic subdural hematoma to determine the incidence and type of gait disturbance. All were pathologically documented cases of frontoparietal chronic subdural hematomas. All were seen by a neurologist or neurosurgeon. Twenty cases which had associated disorders such as hydrocephalus, stroke, or Parkinson's disease or in whom gait could not be assessed, were not used. Of the remaining thirty cases, twelve experienced gait disturbance. In eight of these, it was among the chief complaints and in the remaining four, it was noted in the functional enquiry. The gait disorders were of three types which often overlapped in individual patients: ataxic, apraxic, and hemiparetic. None had unilateral limb ataxia or nystagmus, although marked truncal ataxia was noted in two cases.

In three of the twelve patients, gait disturbance was the only symptom. Two of these were thought to have cerebellar lesions, and the third was correctly diagnosed. All three had bilateral subdural hematomas. Two other cases of bilateral subdurals had no gait abnormality.

Gait disturbance is a significant presenting symptom of chronic subdural hematoma and may be the only symptom.

\section{Localization of Cognitive and Language Impairment in Stroke}

Andrew Kertesz, Robert Coates and Wilhelmine Harlock, London

The localization of cognitive dysfunction and aphasia is attempted by correlating computerized tomographic (CT) and isotope scans (IS) with a standardized aphasia battery and nonverbal performance tasks. Stroke patients who were testable were examined on the Raven's Coloured Progressive Matrices (R.C.P.M.) and Western Aphasia Battery (W.A.B.), which also includes tests of drawing, calculation, praxis, reading, writing and block design. All patients had CT, and some also IS scans, within 35 days of neuropsychological testing. Twelve aphasics had CT scan in the first month; 25 after a year from their stroke; 12 in both the acute and chronic state. The lesions were outlined by a radiologist, without knowledge of symptoms. The outlines were overlapped in groups, defined a priori by taxonomic criteria, according to their test scores.

Results indicate that the CT scan is best suited to localize chronic, and the isotope scan acute, infarcts. The evolution of aphasic syndromes is correlated with lesion size and location. Large perisylvian infarcts caused persisting Global aphasia. Destruction of Broca's area often resulted in only anomic aphasia in the chronic state. Ten nondominant cases were localized and the cognitive deficits described.

\section{CHILD NEUROLOGY II \\ Olivo-Ponto-Cerebellar Degeneration in Infancy: Correlation with Clinically Impaired Blink Response}

H. Darwish, H. Alcala, and D. Demelo, Calgary, Houston and St. Louis

The blink response to corneal tactile stimulation has an ipsilateral and a contralateral component. Normally a bilateral response is present when one cornea is stimulated. The finding of a prominent delay and incomplete closure in the blink of the eye contralateral to the stimulus, when the same eye blinks normally to direct (Ipsilateral) stimulus is an unusual clinical finding suggesting dysfunction at the pontine level. Full term newborns with normal neurological examination, showed brisk bilateral response to corneal tactile stimulus. Low birth weight infants who had no active metabolic or respiratory disease at the time of examination showed a high incidence of impairment of the contralateral blink response to tactile corneal stimulus; in the infants with an abnormal blink, the neurological examination was usually abnormal, and $80 \%$ had difficulty in feeding.

A 7 month old infant was admitted for evaluation of recurrent pneumonias and failure to thrive. Low implantation of thumbs, congenital hip dislocation, and a high arched palate were noted. She had a poor alerting orienting response and spontaneous limb movements were infrequent. Prominent variation in tone was present. A persistent moro was noted as well as an obligatory ATNR and deep tendon areflexia. She had bilateral blink response to light but no visual fixation or ocular pursuit. A consistent delay and failure in complete closure was noticed in the contralateral component of the blink response in either eye, to a tactile corneal stimulus. Microscopic examination of the brain stem showed marked demyelination and atrophy of all the main ascending and descending pathways including the descending root of $V$. All cranial nerves showed loss of fibers and demyelination. The inferior olivary nuclei were not discernible. The cerebellum showed generalized atrophy of the external granular, and the molecular layers. The Purkinje cells were subtotally absent. These findings are consistent with "system degeneration" most closely resembling the adult type of olivo-ponto cerebellar degeneration.

\section{Laryngeal Stridor, Lower Brainstem Dysfunction and the Amold-Chiari Malformation}

C. Matwijecky and F. A. Durity, Vancouver

The association of Arnold-Chiari Malformations, meningomyeloceles and hydrocephalus is well known. It is the Type 2 Arnold-Chiari malformation that is of concern in pediatric age group. Signs and symptoms of lower cranial nerve palsies, lower brainstem and cervical cord dysfunction have only recently become appreciated. Laryngeal stridor due to paralysis of vocal cord abductors is an important early ominous sign of tenth cranial nerve dysfunction and is indicative of impending disaster as respiratory arrest could occur at any point in time when stridor is present.

We reviewed all our cases of meningomyeloceles at the Vancouver General Hospital and found only six cases out of fifty-seven that had laryngeal stridor. Two of these died without undergoing surgery. Four patients underwent posterior cervical decompressive laminectomies. All had had previous ventriculoperitoneal shunts for hydrocephalus with no relief of stridor. Three of the four had dramatic relief of stridor following decompression. One of four had surgical decompression for lower brainstem dysfunction and it is too early to assess the effects of surgery.

Since stridor places the child at risk of respiratory arrest, we advocate early recognition of the seriousness of this condition and early surgical decompression. Children showing signs of stridor should be monitored closely both pre- and post-operatively in the pediatric Intensive Care Unit.

Bone Age in Children with Minimal Brain Dysfunction G. Schlager, D. E. Newman, H. G. Dunn, J. U. Crichton and M. Schulzer, Vancouver

Skeletal maturation has seldom been studied in minimal brain dysfunction (MBD). Relevant studies have compared bone age to radiological norms but not to those of the local population.

Sixty children ( 45 boys, 15 girls) diagnosed in a multi-disciplinary clinic as having MBD were assessed for bone age according to Greulich-Pyle standards. Ten had received stimulants but only for short periods. Six (10\%) were found to have a significantly retarded bone age, whereas only one had a significantly advanced bone age. Thirty eight had a bone age below, and 13 above, the Greulich-Pyle mean line.

The bone age of 58 of these children with MBD was re-assessed by the same radiologist without knowledge of the child's chronological age. The mean bone age was found to be more than 8 months lower than the mean chronological age. A control group of 58 children ( 38 boys, 20 girls) with trauma or idiopathic scoliosis was assessed in an analgous manner and also had a lower mean Greulich-Pyle bone age than chronological age, but the difference was only 3.4 months. 
Analysis of co-variance was applied to the lines indicating bone age vs. chronological age on a logarithmic scale for each category. The following conclusions emerged:

(1) There is a trend for the mean bone age of children with MBD to be lower than that of "normal" control children in our population, but the difference is not statistically significant.

(2) When the data from our study and control subjects are combined into a common slope, this is significantly delayed in its intercepts as compared to Greulich-Pyle norms.

(3) Children with MBD exhibit significantly greater variance about the mean regression line than control subjects; it is therefore possible that a subgroup of MBD children may have special characteristics including low bone age.

\section{Coma Following Cardiorespiratory Arrest (CRA) in Childhood}

\section{S. S. Seshia, K Sankaran and P. N. Chow, Winnipeg}

The data in 26 patients comatose after resuscitation from CRA were analysed. All were referred to the Pediatric Neurology Service, Children's Centre, and were examined with 12 hours of resuscitation. The mean duration of arrest was 10.9 minutes in those who developed CRA in, and 27.2 minutes in those who "arrested" outside, the Centre. Factors preceding and contributing to CRA included, (1) metabolic acidosis - 7 cases, (2) hypoxia

13 cases, (3) hypercapnea - 3 cases, (4) parenteral administration of Diazepam - 4 cases and Phenobarbital Sodium - 2 cases. Depending on the change in the neurological status from the time of initial examination, cases were divided into 3 groups: (1) those who showed no change - 8 cases, (2) those who improved - 8 cases, (3) those who deteriorated - 10 cases. 18/26 patients $(69 \%)$ died and of the survivors, $3(11 \%)$ were normal at discharge. There was a significant relationship between the following clinica variables (as at the first examination) and outcome: (a) coma severity, (b) cye findings, (c) motor patterns, (d) disturbed temperature regulation, and (e) inability to maintain blood pressure. We suggest: (1) the outcome is poor in children comatose after resuscitation from CRA, (2) the assessment of the above mentioned clinical variables within 12 hours of resuscitation, provides significant prognostic information, (3) recognition of factors predisposing to CRA may reduce the incidence of CRA and (4) pediatric cardiopulmonary resuscitation programs must stress the importance of restoring effective cerebral circulation within 4 minutes of CRA (Stephenson Jr. 1974).

\section{ELECTROPHYSIOLOGY II \\ Position and Phase Dependence of Crossed Reflexes}

L. Gauthier and S. Rossignol, Montreal

A noxious electrical stimulus train applied to a cutaneous nerve of a hindlimb (superficial peroneal) can evoke extensor or flexor responses in the contralateral limb. This reversal of reflexes was observed in two conditions: when changing the initial limb position and during locomotion.

In acute spinal cats (Th13) injected with Clonidine, extensor responses occur in the contralateral limb when it is placed by hand in flexion. However, when the limb is extended, crossed flexion is evoked while crossed extension is abolished (Grillner and Rossignol, Brain Research, In Press). Further experiments have led to the conclusion that crossed extensor responses appear unless flexor muscles are stretched, in which case crossed flexion occurs (Rossignol, Neuroscience Abs 3:277, 1977).

Similar stimuli were delivered in various phases of the step cycle in mesencephalic and thalamic cats walking on a treadmill. Crossed flexion and crossed extension responses were observed during different parts of the cycle. If cycle durations are normalized to 100 , beginning and ending at the onset of the flexor burst in the contralateral limb, $85 \%$ of flexor responses observed occurred when the stimuli were given in the period covering the last $15 \%$ and the first $35 \%$ of the cycle. This period includes the end of the stance phase and about $2 / 3$ of the flexion phase. Similarly, $85 \%$ of crossed extensor responses occurred in the remainder of the step cycle i.e. during most of the stance phase. It should be emphasized that crossed flexion can occur even before the onset of the flexor EMG burst and be absent in the later part of the burst. This suggests that the response pattern is not determined by the presence or absence of EMG. Rather, it appears that cross flexion is evoked when the flexors are stretched at the end of the stance phase and beginning of the swing phase. This would be in accordance with the findings that, in static conditions, the state of stretch of flexor muscles exerts a control over the appearance of crossed flexion responses.

\section{Practical Use of The Ring Finger In Sensory Conduction For The Early Carpal Tunnel Syndrome (Camel Test) \\ Charles A Simpson, Victoria}

Brandstater, at the 1972 Canadian Congress of Neurological Sciences, showed the value of the difference between median and ulnar antidromic sensory latency at the ring finger in the carpal tunnel syndrome (CTS). Orthodromic conduction at the ring finger in 203 limbs with clinical CTS confirmed this and also often gave a visual confirmation with a sensory nerve action potential having one hump (normal Dromedary) or two humps (abnormal Bactrian). Hence the name Camel Test. Thirteen percent of these limbs which were abnormal would have been considered normal by conventional sensory testing. Thus this test is useful in confirming abnormality in the $15 \%$ of patients with CTS who are generally considered to have normal findings on conventional testing. Tests on 34 normal control limbs gave a median-ulnar peak latency difference at the ring finger $(\mathrm{Pm}$ $\mathrm{Pu}$ ) of $0.13 \pm 0.25 \mathrm{~m}$. sec. Sixty three nonCTS limbs gave a $\mathrm{Pm} \cdot \mathrm{Pu}$ of $0.09 \pm 0.35 \mathrm{~m}$. sec. Therefore $\mathrm{Pm}-\mathrm{Pu}$ values of $0.5 \mathrm{~m}$. sec. or more were considered abnormal. Forty two percent of patients with clinical and electrical CTS on one side had abnormal $\mathrm{Pm}-\mathrm{Pu}$ values on the opposite asymptomatic limb. The test is practical, simple, sensitive, has its built in temperature control (median vs. ulnar in the same finger) and distance measurement is not critical. Some of the difficulties and possible sources of error are discussed.

\section{Conduction Time Through Brain Stem to Cortex Andrew Eisen and John Stewart, Montreal}

The blink reflex (J. Kimura, Brain 98: 413, 1975), and auditory evoked response (J. J. Stockard, et al, Mayo Clin. Proc. 52: 761, 1977) are two physiological tests of proven value in localizing brain stem disease. This study describes a method of measuring conduction from the brain stem to the cortex using somatosensory responses.

The cervical (SEPC) and cortical (SEPA) somatosensory responses were simultaneously recorded over the $C_{7}$ spinous process, and contralateral cortex respectively. The responses were evoked by unilateral median nerve stimulation in 45 healty volunteers aged $15-63$ years (mean 33.3 ). The mean onset latencies of both responses measured from the initial negative (upward) deflection were $10.7 \pm 1.0 \mathrm{~ms}$ (range $8.5-12.4 \mathrm{~ms}$ ), and $15.9 \pm$ $1.2 \mathrm{~ms}$ (range $13.8 \cdot 18.6 \mathrm{~ms}$ ) respectively. Both responses showed a highly linear correlation with arm length, given by $y=0.16 x+0.15(r=0.87)$ for SEPC, and $y=0.18 x+3.52(r=0.83$ for SEPA. The conduction time from the $C_{7}$ spinous process to the contralateral cortex (TB) given by SEPA - SEPC was $5.1 \pm 1.05 \mathrm{~ms}$ (range $3.0-7.2 \mathrm{~ms}$ ).

In ten subjects a comparison was made of the latencies of SEPA and SEPC as well as TB derived from right versus left-sided stimulation. The mean differences were: $0.51 \pm 0.42 \mathrm{~ms}$ (SEPA); $0.4 \pm 0.3 \mathrm{~ms}$ (SEPC); and $0.58 \pm 0.33 \mathrm{~ms}(\mathrm{~TB})$. The allowable differences of right versus left-sided stimulation (less than $2.5 \mathrm{SD}$ of mean) being $1.6 \mathrm{~ms}, 1.2 \mathrm{~ms}$ and $1.4 \mathrm{~ms}$ respectively.

These measurements should prove useful in detecting or confirming disease affecting levels of the neuroaxis between the cervical cord, brain stem and cortex when clinically suspected but not demonstrable. Since SEPA and SEPC are obtained by the same stimulus, their latencies would be equally prolonged in disease of the peripheral nervous system, and thus prolongation of TB would only occur as a result of central nervous system disease.

\section{Human Far-field Auditory and Somatosensory Evoked Potentials}

\section{T. W. Picton and J. F. Seguin, Ottawa}

Early components of the auditory and somatosensory evoked potential, generated in subcortical portions of the sensory pathways, can be recorded using averaging techniques from surface electrodes. In the first 10 milliseconds after a moderately loud click ( $80 \mathrm{~dB}$ above normal threshold) a characteristic sequence of waves can be recorded from vertex to mastoid electrodes. Wave 1 at $1.70 \mathrm{~ms}$ is generated by the cochlear nerve fibers, waves II and III at $2.78 \mathrm{~ms}$ and $3.83 \mathrm{~ms}$ by regions of the pons, waves IV and V at 5.08 and $5.58 \mathrm{~ms}$ from regions of the mid-brain, and wave VI at $6.95 \mathrm{~ms}$ possibly from thalamic areas. In response to electrical stimulation of the median nerve at the wrist at an intensity $20 \%$ above thenar twitch threshold, a series of four waves can be recorded prior to the cortical evoked potential from scalp to shoulder electrodes. Wave I at $9.05 \mathrm{~ms}$ is 
generated in the region of the brachial plexus, wave 2 at $11.21 \mathrm{~ms}$ from the spinal nerve roots or dorsal columns, wave 3 at $12.68 \mathrm{~ms}$ from the brainstem (possibly the medullary nuclei) and wave 4 at $13.49 \mathrm{~ms}$ from the thalamus. These early evoked potential components have very precise latencies and morphology. In patients, such recordings can therefore be used to localize and evaluate abnormal function in the auditory and somatosensory pathways. These abnormalities are most appropriately demonstrated by measuring the latencies and amplitudes of each component relative to the others, and by assessing the symmetry of these measurements in the responses to right and left stimuli.

\section{NEUROBIOLOGY III (Vascular)}

\section{Successful Production of a Guinea Pig Stroke Model}

Daniel S. Heffez and William Sheremata, Montreal and Miami

Guinea pigs are ideal laboratory animals for a large variety of studies, but investigators heretofore have failed to develop a guinea pig stroke model. Because of the relevance of such a model to immunological and other studies, we undertook this task.

A preliminary study of 12 animals failed when carotid ligation alone was carried out. However surgical isolation of the internal carotid followed by embolization of $20 \mathrm{mgm}$. of sephadex microspheres produced seizures, hemiparesis, and piloerection of the contralateral side in 38 of 48 animals who survived the procedure. Cerebral infarction occured in 80 percent of animals studied pathologically. None of 50 unoperated animals, nor any of 8 animals who were sham operated demonstrate any clinical symptoms. 13 of the survivors died between the 6th and 13th day after operation. The clinical picture following embolization resembled the human following an embolic event, and the pathological picture was that of hemorrhagic infarction as seen in the human.

Embolic crebral infarction in the guinea pig can be predictably obtained following isolation of the internal carotid artery and embolism with sephadex microspheres. Such a model will be of great value in studying immune responses to release of nervous system antigens as well as the study of physiological responses to sudden deprivation of cerebral blood flow.

\section{The Use of Intravital Dyes As A Marker For Ischemic Neurons}

F. A. Durity and M. J. Kendall, Vancouver

In a study of global ischemia in the Mongolian gerbil, it seemed that identification of the locus of injury inflicted might be highlighted by use of the fluorescent dyes, Evans Blue and Procion Yellow. Animals were anaesthetized with intra-muscular ketamine and subjected to bilateral carotid ligation for 10-15 minutes. Circulation was re-established and animals maintained up to 24 hours. $0.4 \mathrm{mg} / 100 \mathrm{gm}$. of $2-3 \mathrm{M}$ Ureaphil was then injected into right carotid artery in some, to facilitate opening of the blood-brain barrier and followed within 10 minutes by $0.4 \mathrm{ml} / 100 \mathrm{gm}$. of a $4 \%$ solution of either of the above dyes. Animals were decapitated within a half-hour, the brains examined grossly for evidence of staining, then fixed in formalin and examined histologically for the presence and location of fluorescence.

In 16 control animals fluorescence was detected only in the blood vessels of the injected hemisphere. Five animals subjected to ischemia and recirculated up to 24 hours, but not given Ureaphil, similarly revealed only intravascular fluorescence.

Those animals $(n=24)$ subjected to ischemia for $10-15$ minutes, recirculated up to 24 hours and subjected to intracarotid Ureaphil followed by intravital dye injection showed fluorescence within ischemic neurons provided recirculation time exceeded 18 hours. These neurons were located in the classical areas of vulnerability, including hippocampus and deep layers of cerebral cortex. Neuronal membranes seemed to remain relatively intact up to 18 hours following ischemia.

\section{Quantitative Estimate of Pinocytosis In Acute Experimental Hypertension}

\section{S. Nag, D. M. Robertson and H. B. Dinsdale, Kingston}

Cerebral cortical arterioles become permeable to horseradish peroxidase (HRP) during episodes of acute experimental hypertension. Our previous studies of hypertensive animals showed that pinocytosis appeared to be the principal mechanism for the increased blood-brain barrier permeability observed. The purpose of these experiments was to obtain a quantitative index of pinocytotic activity to determine whether enhanced pinocytosis was confined to the permeable arteriolar segments of hypertensive animals, and also to determine whether HRP itself affected the pinocytotic index as suggested by some workers. HRP was injected intravenously in 12 rats which then received an angiotensin infusion lasting $1 \frac{1}{2}$ minutes following which the animals were perfusion fixed. Control animals received saline only or HRP only. The surface area of arteriolar endothelium in cross section was determined by a planimeter from overlapping electron micrographs taken at a constant magnification around the circumference of the vessel wall. Results indicate a significant ( $P$ less than .001 ) increase in the number of pinocytotic vesicles in the permeable arteriolar segments of hypertensive animals as compared with non-permeable arteriolar segments of the same animals and comparable segments of normotensive rats. HRP alone did not affect the pinocytotic index, there being no difference ( $P$ greater than .05 ) in the number of vesicles in normotensive animals receiving saline only and those receiving HRP only. Our previous observation that disruption of endothelial cells or their tight junctions did not occur, was confirmed. (Supported by Grant MRC MT1647).

\section{Lysis of Blood Clot In The Experimental Treatment of Subarachnoid Hemorrhage}

Eric W. Peterson, Sin Huat Choo and A. J. Lewis, Ottawa

Blood clot following subarachnoid hemorrhage lies in close contact with the cerebral blood vessels and is considered to be related to vasospasm. If an aneurysm is successfully clipped, lysis and removal of clot could avoid this complication. In acute experiments on twenty adult cats with controlled ventilation and monitoring of blood pressure and blood gases, subarachnoid hemorrhage was produced by the injection of blood into the basal subarachnoid space using a small silicone catheter. After an hour, when firm clot had been produced, the space was irrigated through the same catheter using Streptokinase-Streptodornase (Varidase ${ }^{(k)}$ ) in $10 \mathrm{ml}$ of Elliott's B solution, over a period of one hour. The cats were sacrificed three hours after the irrigation and the brains were perfused and subjected to gross and microscopic examination. Successful lysis of the clot was demonstrated and there were no histopathological changes in the brains which could be attributed to this treatment. It is felt that this may offer an approach to the problem of subarachnoid hemorrhage and the complicating vasospasm by removing its cause and thereby permitting earlier operation for the aneurysm and limiting the danger of re-hemorrhage.

\section{Treatment of Induced SAH with Sodium Nitroprusside and Phenylephrine in the Spontaneously Breathing Primate}

Charles Rothberg, Bryce Weir, Thomas Overton and Mike Grace, Edmonton

The effects of simultaneous intravenous administration of sodium nitruprusside and phenylephrine on post subarachnoid hemorrhage $\mathrm{rCBF}$, vessel calibre and survival were studied in the spontaneously breathing cynomolgus monkey. We have previously documented reduced $\mathrm{rCBF}$, cerebral vasospasm, respiratory irregularities, hyposemia and poor survival as the responses to induced surbarachnoid hemorrhage (SAH) in the spontaneously breathing cynomolgus monkey.*

The rationale of this form of treatment has been outlined by George Allen: the universal vascular smooth muscle relaxing effect of sodium nitroprusside and the selective constrictor effect of phenylephrine on noncerebral vascular beds. This treatment is directed at improving post-SAH $\mathrm{rCBF}$ and vasospasm, and subsequently the quality of survival.

A total of eleven monkeys was used. Eight received an induced SAH of autogenous blood $(1.67 \mathrm{ml} / \mathrm{kg}$ body weight) and were subsequently treated with simultaneous intravenous sodium nitroprusside and phenylephrine. The remaining three animals did not receive a hemorrhage, but served as a control group and were treated with the nitroprusside and phenylephrine as if they did receive an SAH.

Regional cerebral blood flow was measured using the 133 Xenon clearance technique and the "initial slope" analysis. The nitroprusside and phenylephrine were administered in dosages to maintain the mean arterial blood pressure at pre-SAH levels. 
The following table illustrates the post-SAH rCBF ( 6 flows) and intradural internal carotid artery diameter (IDICA) expressed as a percentage of the pre-SAH values for both untreated and treated SAH groups.

Control values are also included

Post-SAH rCBF and IDICA

\begin{tabular}{lllllllll}
\hline GROUP & 1 & 2 & IDICA & 3 & 4 & 5 & 6 & IDICA \\
\hline "SAH & $40 \pm 6$ & $61 \pm 11$ & $70 \pm 4$ & $77 \pm 23$ & $66 \pm 15$ & $64 \pm 10$ & $60 \pm 8$ & $80 \pm 4$ \\
SAH \& & & & & & & & & \\
Treatment & $44 \pm 6$ & $56 \pm 4$ & $68 \pm 4$ & $58 \pm 6$ & $55 \pm 7$ & $51 \pm 6$ & $50 \pm 6$ & $80 \pm 7$ \\
Control & $95 \pm 4$ & $82 \pm 6$ & $102 \pm 4$ & $91 \pm 9$ & $82 \pm 9$ & $86 \pm 7$ & $80 \pm 8$ & $103 \pm 1$ \\
\hline
\end{tabular}

$\pm=$ S.E. M. (standard error of the mean)

Treatment of induced SAH in the spontaneously breathing cynomolgus monkey with sodium nitroprusside and phenylephrine did not improve post hemorrhage rCBF, vessel calibre or survival.

\section{Influence of Arterial $\mathrm{PCO}_{2}$ on Vessel Calibre and Response to Adenosine of Cat Pial Arterioles}

Donald P. J. Boisvert, Peter C. Gregory and A. Murray Harper, Edmonton

The television image-splitting technique was used to study the influence of arterial $\mathrm{pCO}_{2}$ on the dilatatory response of pial arterioles to topically applied adenosine in thirteen chloralose anesthetised cats. The effect of altering $\mathrm{pCO}_{2}$ on pial arterial calibre was studied in seventeen cats.

The arterioles dilated with hypercapnia and constricted with hypocapnia. Over the range of arterial $\mathrm{pCO}_{2} 15-55 \mathrm{mmHg}$ the response fitted a linear regression: arterioles less than 100 diameter $-0.96 \%$ per $\mathrm{mmHg}$ change in $\mathrm{pCO}_{2}$. There was no evidence of any reduction in $\mathrm{CO}_{2}$ reactivity at extreme hypocapnia suggesting that failure of cerebral blood flow to decrease further at $\mathrm{pCO}_{2}$ less than $25 \mathrm{mmHg}$ is not due to maximal arteriolar constriction.

Perivascular injection of $10-{ }^{5} \mathrm{M}$ adenosine produced a pial arteriolar dilatation of $29.2 \pm 2.7 \%$ (S.E.M.) at normocapnia $\left(\mathrm{pCO}_{2}\right.$ approximately equal to $35 \mathrm{mmHg})$. During hypocapnia $\left(\mathrm{pCO}_{2}\right.$ approximately equal to 25 $\mathrm{mmHg}$ ) the adenosine response was reduced by $50 \%$ (p less than 0.001 ). During hypercapnia $\left(\mathrm{pCO}_{2}\right.$ approximately equal to $48 \mathrm{mmHg}$ ) the adenosine response was reduced by $71 \%$ ( $p$ less than 0.001 ). All adenosine responses were independent of initial arteriolar diameter.

These results indicate that adenosine reactivity is markedly decreased by the changes in perivascular $\mathrm{pH}$ and vascular resistance resulting from moderate changes in arterial $\mathrm{pCO}_{2}$. Therefore, adenosine is unlikely to play a key role in the vasodilatation resulting from cerebral ischemia.

\section{NEUROSURGERY V (Spinal II) Spinal Epidural Abscess}

J. Elliott and G. B. Thompson, Vancouver

Eight cases of spinal epidural abscess were reviewed, from 1955 to 1977 , at the Vancouver General Hospital. Patients varied in age from seventeen to seventy-two years, and in sex, including two females and six males. The presentations were acute in all cases and showed a characteristic progression of spinal ache, root pain, weakness and finally paralysis. Diagnosis was made on the basis of history, clinical findings, lumbar puncture and myelography where feasible. There was one sterile abscess, and in seven cases the causative organism was Staphylococcus aureus. The sources of infection were: hematogenous, spread from skin lesions, and osteomyelitis; the former being associated more with thoracic and lumbar lesions, the latter with cervical lesions. Locations of the abscess varied from cervical to lumbrosacral, and from anterior to posterior epidural spaces.

Treatment in all cases consisted of operative decompression with prolonged antibiotic coverage, with or without steroid therapy.

The outcome in all cases correlated with the degree and duration of preoperative deficit. It was concluded that spinal epidural abscess represents a potentially curable cause of significant neurological deficit when the diagnosis is made early before prolonged deficit has occurred and operative treatment is carried out urgently.

\section{Management of the Traumatic Neurogenic Bladder in Males}

N. W. Struthers, W. S. Tucker, W. J. Horsey, A. R. Hudson, J. M. Houston and R. McCallum, Toronto

A catheter-free state is the aim of treatment in the traumatic neurogenic bladder. Transurethral sphincterotomy, or resection of the bladder neck are the procedures advocated to reduce outflow resistance but the choice of operation and timing remain the subjects of controversy.

Over the past 6 years at St. Michael's Hospital, the following bladder management programme has been used. During the period of the patient's care in the Neurosurgical service the bladder is drained using either a Foley or Gibbon catheter connected to a closed system. Following transfer to the rehabilitation unit intermittent catheterisation is established. Failure to void within 2 weeks, or unacceptably high residual urines at a month warrant a full urological investigation. This includes the dynamic retrograde cystourethrogram (D.R.C.) which is a radiographic technique for producing controlled overflow voiding. Demonstration of sphincter dysynergia by the D.R.C. is regarded as an indication for early transurethral external sphincterotomy. This permits the rapid establishment of satisfactory bladder emptying, and avoids the problems associated with detrusor muscle hypertrophy and secondary bladder neck obstruction which have necessitated bladder neck resection in the past.

65 patients (34 quadriplegics and 31 parapalegics) have been rehabilitated with an average hospital stay of 130.6 days. 17 patients have had sphincterotomy alone, 9 patients sphincterotomy with prostatic resection, and 2 patients prostatic resection. Bladder neck resection, as a sole procedure, was not required. 3 patients in the entire series have permanent catheters, one having refused sphincterotomy. 26 of the 28 operated patients are voiding without a catheter.

\section{The Surgical Treatment of Spinal Metastases Causing Compression}

R. G. Perrin and K. E. Livingston, Toronto

Spinal metastases causing cord compression occur in about $5 \%$ of patients with systemic cancer. They inevitably produce devastating morbidity and it is widely held that surgical treatment is ineffective. In our experience such a gloomy verdict is not justified.

We have treated 100 patients with spinal metastases causing cord or cauda compression between 1968 and 1978. In seven of these, cord compression was the result of pathological fracture.

Thirty-nine men and 61 women ranged in age from 21 years to 80 years. Thirty percent (all women) had breast cancer. The most common primary in men was prostatic carcinoma.

Pain was the earliest and most prominent symptom, followed by weakness. Pre-operatively 97 patients demonstrated motor impairment - 50 were completely bedridden, and 21 were totally paraplegic. Sensory abnormalities were defined in 90 patients. Five presented with Brown-Sequard syndrome. Bladder dysfunction was recorded in 40 patients.

Spinal compression occurred in the thoracic region in 76 patients. Surgical treatment involved urgent and extensive laminectomy decompression. Concomitant spinal stabilization was performed in ten cases, involv. ing posterior rib graft fusion (7) and Harrington rod instrumentation (3).

January 1978, 29 of these patients were living. The average postoperative survival was 2.3 years. Seventy one patients had died with an average survival of 8.8 months. Surgical decompression produced effective pain relief in $70 \%$.

Fifty-eight patients were ambulatory post-operatively - 40 were walking and continent of urine 6 months following surgery (including 5 patients who presented totally paraplegic.)

A positive approach and aggressive management, in this problem, can achieve results superior to those generally reflected in the literature.

\section{A Brief Resume of the Natural History of Spinal Cord Injury W. O. Geisler, Toronto}

An earlier epidemiological study of the natural history of spinal cord injury in Ontario reveals the peak incidence to be on a week-end, in the summer, late in the afternoon or early evening, in the second decade of life and victim likely to be male. Over two-thirds of the victims were injured in motor vehicle accidents, falls or diving accidents. 14.3\% of a group of 224 patients showed progressive neurological change. It seems reasonable that a strong programme of public education concerning the dangers of diving and driving and the advantage of industrial safety and early effective immobilization would be rewarding. 
A retrospective review spanning twenty-nine years and including the histories of 1501 traumatic spinal cord injured patients revealed renal failure to be the predominant cause of death. Comparing death in these patients with the leading causes of death in the general population, (C. V. Disease, Cancer, Respiratory Disease, Accidents and Suicide), the study revealed an increase in younger age groups in death from cardiovascular disease, an increase in older age groups from death due to respiratory disease and in both young and old groups, an increase in death due to suicide and cancer. Special mention of the cancer relation will be made. There has been no improvement in Life Expectancy for the spinal cord injured in the last ten years. New ideas in prevention and treatment must be forthcoming.

\section{NEUROSURGERY VI}

\section{Early Neurosurgical Procedures in Craniofacial Dysostosis}

\section{Harold J. Hoffman, Toronto}

The human brain grows more rapidly during the first six months of life than it does during the remainder of childhood. In the case of synostosis of a cranial suture, this tremendous growth of brain carries on but must find a fresh direction in which to expand. Compensatory growth occurs in the opposite direction to that of the synostosed suture.

Closure of the coronal suture is usually associated with closure of the frontosphenoidal and frontoethmoidal sutures in the floor of the anterior cranial fossa. The ventral part of the frontal lobes establish the size and alignment of the floor of the anterior cranial fossa which in turn determines the extent and direction of facial growth. Patients with Crouzon's disease and other forms of craniofacial dysmorphism have closure of their coronal sutures in association with closure of the frontosphenoidal and frontoethmoidal sutures. A linear craniectomy along the coronal suture does nothing for the synostotic process at the base of the skull. On the other hand, if one opens the basal skull sutures at the same time as the calvarial sutures, one allows for the expanding frontal lobe to move the frontal bone into normal position and thus expand the anterior cranial fossa as well as allowing for future growth of the anterior cranial fossa. We have used this technique in eight infants with craniofacial dysmorphism and feel that these children will not require a major craniofacial repair in the future as a result of adequate repair of their craniosynostosis early in infancy.

\section{Posterior Interosseus Nerve Injury}

\section{Alan Hudson, J. Waddell and R. McBroom, Toronto}

Patients suffering from posterior interosseus nerve palsy exhibit a characteristic clinical picture. Muscles supplied by the radial nerve allow extension of the wrist with radial drift, but extension of the fingers and thumb is hampered by paralysis of the muscles supplied by the posterior interosseus nerve.

Ten cases are presented. Two suffered from spontaneous entrapment. Three sustained injuries during surgical procedures. A $16 \mathrm{~mm}$. film clip illustrates the surgical anatomy and approach to the nerve in the antecubital fossa and on the extensor aspect of the forearm. Two cases in which interfascicular nerve grafts were used are described.

The purpose of the presentation is to illustrate points of surgical anatomy and technique which the authors consider important in the management of these patients.

\section{Brachial Plexus Injuries: Clinical and Electrophysiological Observations W. Becker, P. M. Richardson, A. J. Aguayo, L. P. Renaud and E. Matthew, Montreal}

Fifteen patients with severe blunt trauma to the brachial plexus were followed from six months to ten years with repeated clinical and electrophysiological examination. Ten of the injuries were associated with motor vehicle accidents, one with a falling weight, two with shoulder dislocations and two with prolonged coma and presumed nerve compression.

In one patient, with directly observed disruption of the infraclavicular portion of the brachial plexus, sensory action potentials at the wrist and muscle action potentials at the hand were measured daily from the fifth to the ninth day after injury. Sensory action potentials with normal conduction velocity but progressively decreasing amplitude were recorded until the eighth day. Muscle action potentials with nearly normal conduction velocity and small amplitude persisted until the ninth day. In two patients examined in the week after injury, nerve stimulation at the elbow evoked a distal muscular response but stimulation at Erb's point did not. After one week, neither stimulus was effective. In twelve paralysed and anaesthetic limbs, median and ulnar sensory action potentials were evaluated between one month and two years after injury. They were absent except for one median and one ulnar nerve. Three years after the injury, sensory action potentials had reappeared in some patients.

Two patients with early recovery of hand function, had a residual upper brachial plexus palsy. Ten patients with extensive distal denervation of muscle and loss of sensation fared poorly despite the gradual return of some proximal muscle function. Three patients with less initial sensory loss recovered much better.

\section{Cerebellopontine Angle Meningiomas Michael Schwartz, Toronto}

In the past thirty months the author has operated upon eleven patients with cerebellopontine angle tumors, of which, surprisingly, four were meningiomas. These tumors were distinct from the acoustic schwannomas in their clinical presentation, in that three had ataxia as a major symptom and each had trigeminal signs out of proportion to the hearing loss. Three patients had typical sensorineural hearing loss and all had nystagmus. Three of the tumors had a distinctive lobulated appearance on the CT scan and were recognized as meningiomas prior to surgery. The tumors were approached by the suboccipital route.

Complete excision was achieved in two cases, subtotal excision in one, and biopsy only in the other. Tumor consistency and site of origin rather than size governed the completeness of resection. The softest tumor which engulfed neural structures rather than displacing them proved the most difficult. In this patient, considerable improvement in ataxia, her chief complaint, was achieved and maintained to the present (19 months) by extensive bony decompression. In another patient, a clivical origin precluded complete excision. The facial nerve was spared in every case. A partial fifth nerve deficit was produced in one and a temporary glossopharyngeal and vagus palsy in another. All patients have returned to their preoperative activities. With the microscope and an aggressive approach, extensive or complete resection is often possible, even in large tumors, without the production of unacceptable morbidity.

\section{Unusual Intracranial Developmental Lesion}

B. Curry, A. W. F. Fisher and C. W. Taylor, Calgary

An unusual cerebello-pontine angle tumor is described in a 43 year old patient who presented following a minor head injury, and having a history of total deafness in the right ear since childhood. The lobulated tumour proved to be a rather fatty hetertopic salivary gland containing a microscopic adenoid-cystic carcinoma. Only two previous cases of intracranial salivary gland tissue (both in the pituitary fossa) have been described. This is the first in the cerebello-pontine angle. Its uniqueness is accentuated by the presence of the primary salivary gland tumor. Mechanisms to account for its presence in this site are discussed.

\section{Chronic Cerebellar stimulation in Cerebral Palsy}

E. C. G. Ventureyra and L. P. Ivan, Ottawa

The purpose of this study is to assess the value and feasibility of chronic cerebellar stimulation in patients suffering from cerebral palsy. Between July and November 1977, eleven patients were treated at the Children's Hospital of Eastern Ontario by implanting cerebellar electrodes for chronic cerebellar stimulation. Ten patients were suffering from cerebral palsy of the spastic athetoid type and one from post-traumatic spastic hemiplegia. The age ranges were between 3 to 27 years, the I.Q. within normal limits.

One pair of electrodes was placed in each patient on the anterior surface of the cerebellum in the paravermal area biocalibrated in the operating room with a setting of $0.5 \mathrm{msec}$., 1 milliamper, 200 CPS, 7 minutes on, 7 minutes off cycle. The patients were functionally assessed one week, three months and six months after surgery using speech, respiration, muscle tone and activities of daily living as parameters.

Seven patients showed significant degree of muscle relaxation which permitted them to develop new motor skills. Three patients remained unchanged functionally after the insertion of the cerebellar stimulator. One patient became worse after surgery. Changes in the respiratory pattern were found in most patients during stimulation. Drooling of saliva improved in some cases, the sleep pattern was altered in others. No surgical complications were observed except for one patient who developed a 
seroma at the receiver site which later showed staphylococcus albus colonization requiring removal of the receiver and vigorous treatment with antibiotics. In spite of discontinuing the stimulation, this patient did not lose his functional gains.

This pilot study yielded a number of useful informations that helped us to develop criteria for patient selection and permitted the design of a modified surgical technique that makes the procedure safer and easier. It is our impression that for certain patients, chronic cerebellar stimulation provides valuable help in terms of functional improvement. This method should be further explored using a rigid protocol for functional evaluation.

\section{CEREBROVASCULAR III (Measurement and Management) Pulmonary Edema Following Fatal Aneurysm Rupture Bryce Weir, Edmonton}

A retrospective clinical-pathological analysis of 78 cases of fatal subarachnoid hemorrhage (SAH) was carried out. $71 \%$ had a pathological diagnosis of pulmonary edema (PE) and of these, $31 \%$ had a clinical diagnosis of PE. 55 cases with pathologic $P E$ had a mean age of $47 \pm 14$ years. 32 of these died on the first day following their SAH. The average survival, SAH to death (days) in this group was $10 \pm$ days. For 23 cases without pathological evidence of PE the mean age was $52 \pm 17$ years. 19 died on the first day post SAH. The incidence of PE fell with the passage of time following SAH while the occurrence of pneumonia and embolism increased. The incidence of subarachnoid hemorrhage, intracerebral, intraventricular hemorrhage, mid-brain hemorrhage, subdural and extradural hemorrhage was not significantly different in the groups with pathological PE and those without. $46 \%$ of the patients with pathologic PE had middle cerebral aneurysms while $33 \%$ of the patients without pathological PE had aneurysms at that site. This difference was not statistically significant and neither was the incidence at other aneurysmal locations. $92 \%$ of the patients with pathologic PE had cerebral edema while $71 \%$ cases without pathological PE had cerebral edema. For patients surviving 1 to 3 days post SAH $90 \%$ had pulmonary edema, 4 to 14 days following SAH $62 \%$ had pulmonary edema and more than two weeks following SAH only $52 \%$ showed pulmonary edema at autopsy. The incidence of pneumonia showed a reverse pattern being $19 \%$ for those dying 1 to 3 days post SAH and increasing to $50 \%$ for those more than two weeks post SAH. There was hypoxemia and hypocapnia in both groups, more severe in the group that had pathologic PE. The initial blood gases for those dying with pathological PE were $\mathrm{PaO}_{2} 48 \pm 12 \mathrm{mmHg}, \mathrm{paCO}_{2} 27 \pm 9 \mathrm{mmHg}, \mathrm{pH}$ $7.41 \pm .12, \mathrm{O}_{2}$ saturation $85 \pm 14 \%$. Corresponding values for those $\mathrm{dy}$ ing without pathological PE were $62 \pm 9 \mathrm{mmHg}, 32 \pm 5 \mathrm{~mm} \mathrm{Hg}, 7.47 \pm$ .05 , and $92 \pm 5 \%$. The mechanism of pulmonary edema following fatal aneurysmal rupture is felt to be an excessive sympathetic discharge causing increases in pulmonary capillary permeability as well as hypertension within the entire pulmonary tree. Treatment should consist of reducing intracranial pressure, mechanically ventilating patient and possibly employing alpha-blocking agents.

\section{Reversal of Ischemic Deficits by Induced Arterial Hypertension}

N. F. Kassell, S. J. Peerless and C. G. Drake, lowa City and London

Various attempts have been made to reverse ischemic neurological deficits. This report relates the experience with induced arterial hypertension in a series of 21 patients to date with acute, progressive neurological deterioration resulting from ce rebral vasospasm, thromboembolic occlusive disease, and iatrogenic occlusion of major cerebral arteries. Although there were disappointing failures and major complications, the results overall were encouraging. In most instances dramatic temporary or permanent improvement in neurological function occurred. Fifteen patients were permanently and two temporarily improved. No improvement occurred in four instances. The worst results were in patients with vasospasm. There were 5 complications, 2 fatal, which will be discussed. The authors will describe a technique for producing long-term hypertension with vasopressor agents, intravascular hypervolemia and vagal blocking agents.

Role of ICP Monitoring in Neurosurgical Patients

W. J. Peacock, F. Taylor and H. Schutz, Toronto

The intracranial pressure (ICP) was recorded in 203 adult patients over a 5 year period. The ventricular fluid pressure (VFP) was recorded an average of 3.5 days per patient. The infection rate was $1.5 \%$. Patient groups studied were head injuries (53), brain tumours (30), intracranial hemorrhages (24), and a miscellaneous group of 96 patients. These include acquired and congenital hydrocephalus, pseudotumour cerebri, etc. Immediate diagnosis and evacuation of space occupying lesions was always accomplished prior to ICP monitoring. Based on these recordings certain management decisions could be made. We found that ICP monitoring was essential in those brain injuries (34) which required controlled ventilation. In these situations the neurosurgeon is usually completely deprived of customary clinical parameters. Elevated ICP (often unsuspected) was managed by a combination of steroids, Mannitol, glycerin, change in head position, hyperventilation, CSF drainage, surgical decompression and recently barbituate coma. ICP monitoring was useful in the diagnosis and treatment of patients with hydrocephalus and dementia. Bizarre symptoms and signs (false localizing) were observed frequently during plateau waves (Lundberg type A). Unacceptable elevations of intracranial pressure during anesthetic induction was seen initially but careful technique has eliminated these complications. Examples will be presented.

ICP monitoring remains a useful adjunct in the management of many neurosurgical patients.

\section{Regional Cerebral Blood Flow and Extra-Intracranial Arterial} Anastomoses

M. I. Vilaghy, V. C. Hachinski, J. W. Norris, P. W. Cooper and D. W. Rowed, Toronto

When the purpose of the operation is to increase cerebral perfusion, regional cerebral blood flow ( $\mathrm{rCBF}$ ) monitoring may be valuable.

A major problem in assessing the effect of superficial temporal artery (STA) - middle cerebral artery (MCA) anastomoses on rCBF is the artefact created by blood flow in extracranial collaterals. However, by the judicious selection of site and dosage of injection of isotope into the cerebral circulation, these problems are largely overcome. Differentiation of intracranial from extracranial blood flow can be achieved by identifying the individual isotope washout curves.

Thirty rCBF studies were carried out in 8 patients by the intra-arterial method during cerebral arteriography for the evaluation of STA-MCA anastomotic surgery.

Based on these studies, our tentative conclusions are 1) Low rCBF in the absence of hemodynamically significant lesions is not an indication for STA-MCA anastomatic surgery 2) Normal rCBF even in the presence of hemodynamically significant lesions, contraindicates surgery, hence 3) STA-MCA anastomotic surgery for cerebral re-perfusion only has a rationale in patients with hemodynamically significant lesions and low rCBF.

\section{CLINICAL NEUROLOGY V}

\section{A Dominant Syndrome of Hemiplegic Migraine, Nystagmus and Tremor Bengamin Zifkin, Eva Andermann, Trevor Kirkham and Frederick Andermann, Montreal}

A mother and her son suffer from hemiplegic migraine with onset in childhood. Both have nystagmus which has not changed for many years, but the date of onset is uncertain. They have an asymmetrical tremor of the arms, clinically indistinguishable from essential tremor.

Neuro-ophthalmologic examination revealed gross defects in the pursuit movement system. These were: inability to produce smooth pursuit, gazeparetic nystagmus, failure of fixation suppression of the vestibulo-ocular reflexes both horizontally and vertically, rebound nystagmus, and failure to augment the slow phase velocity of optokinetic nystagmus with increasing stimulus velocity. These abnormalities were confirmed by electrooculography, and suggest a disturbance in the connections between the vestibular nucleus and the flocculus of the cerebellum.

Since treatment with propranolol and pizotyline was begun, one patient has had no further episodes of hemiplegia, and the other has had fewer attacks. Tremor was improved in each case.

Hemiplegic migraine has been reported in association with nystagmus, retinal degeneration, deafness and ataxia in varying combinations in three families with autosomal dominant inheritance. These associated neurological manifestations likely represent system degenerations rather than the effect of repeated ischemia imputable to the migraine itself.

The association of two conditions responding to beta blockers in the family presented raises the question of a disturbance in a common biochemical pathway. 


\section{Antibody Forming Cells in Multiple Sclerosis: A Deficiency in Plaque Formation after Polyclonal Activation of B Cells* \\ D. W. Paty and H. K. Cousin, London}

We have been studying a number of immunological parameters in Multiple Sclerosis (MS) patients trying to identify an MS associated immune defect. We have previously reported an MS associated decrease in mitogen responsiveness in HLA A3 \& B7 positive MS patients and a decreased or absent CMI \& humoral response toward herpes simplex virus (type 1) antigen in HLA Dw2 positive MS patients. We now report a survey of antibody producing cells in MS. Peripheral blood lymphocytes (PBL) are separated on ficoll-hypaque and cultured with and without $50 \mathrm{ug} / \mathrm{ml}$ of Pokeweed Mitogen (a polyclonal activator of B cells) at $37^{\circ} \mathrm{C}$ in $5 \% \mathrm{CO}_{2}$ for 7 days. The cells are then washed, corrected for viability, and mixed with sheep red blood cells (SRBC) and complement in Noble Agar with DEAE-Dextran.

Thirty-five normal subjects and neurological controls were compared with 71 patients that meet standard criteria for clinically definite MS. MS patients were not tested during the acute relapse. The mean production of plaques $(\mathrm{P})$ visible to the naked eye was $145 \mathrm{P} / 10^{6} \mathrm{PBL}(\mathrm{SEM}=29.1)$ in the controls and $81 \mathrm{P} / 10^{6} \mathrm{PBL}(\mathrm{SEM}=13.7)$ in the MS patients (P less than 0.05 ). The frequency of non responders (less than or equal to 10 $\mathrm{P} / 10^{6}$ ) was $32 \%$ in the MS group and $17 \%$ in the controls.

This finding of a deficiency in antibody production in a number of MS patients suggests either a " $B$ " cell deficiency or more likely a deficiency in the regulator " $T$ " cell population. We are proposing that there is a defect in regulator " $T$ " cell activity in MS that accounts for the variable finding of high antibody levels to many viruses and low $T$ cell and $B$ cell in vitro activity. This deficiency appears in some assays to be linked to major histocompatability (HLA) markers. Our data on this group of patients is not yet complete. We will be following these patients to re-test them during periods of acute relapse.

* This study was supported by the MS Society of Canada.

\section{A Syndrome of Episodic Hypothalamic Dysfunction: The Kleine-Levin Syndrome Revisited}

Joseph Carlton, Frederick Andermann and Joseph B. Martin, Montreal

In the past 13 years, we have seen 13 patients with the Kleine-Levin syndrome. The outstanding symptom is hypersomia and sleep disturbance, but megaphagia, hypersexuality, abnormal drinking, hallucinations, inappropriate behavior, mood disturbance, singing, amnesia and headache are often just as prominent.

Of 13 patients, 9 were males, five in the second decade, three in the third, and one in the fifth. Four were adolescent females. Two patients were mildly retarded. One patient had a remote traumatic hemiplegia, and in one the syndrome developed following irradiation for a third ventricular tumor. This indicates the possibility of pre-existing neurological abnormality, or even of a symptomatic form of the disorder.

The three young adult males had single attacks with follow-ups of up to nine years. This raises the question of a possibly different etiology in this group, and lesser importance of intrinsic biological cycles.

In girls, the disorder is not as rare as has been suggested. It is often triggered by the menstrual cycle, and may relate to the hypersomnia of pregnancy and menstruation of normal women.

Generalized electroencephalographic abnormalities are the rule, and these are of a type not merely attributable to drowsiness.

An episodic hypothalmic disturbance would account for most of the clinical features, but dysfunction of other limbic structures is probably present as well.

\section{Ocular Motor Function After Cerebral Hemispherectomy}

James A. Sharpe, Alex W. Lo and Hyman E. Rabinovitch, Toronto

Saccadic and smooth pursuit system function was analyzed in five human subjects nine to twelve years after unilateral cerebral hemispherectomy. Surgery had been performed between seven weeks and fifteen years of age for control of epilepsy. Horizontal eye movements were quantitated by infrared reflection oculography and compared with data from fifteen control subjects.

Peak velocities of saccades directed to the side opposite hemispheric ablation were mildly reduced for amplitudes up to $40^{\circ}$ (p less than 0.05 ) in individual patients and saccadic durations were similarly prolonged. Latencies of saccades to $5^{\circ}$ or target steps into the blind hemifield were longer $(\mathrm{p}$ less than 0.01 ) than to steps into the ipsilateral field, as expected. However, saccadic latencies for $20^{\circ}$ and $40^{\circ}$ target steps were similar in both horizontal directions. Differences between saccadic latencies for parafoveal $\left(5^{\circ}\right)$ and eccentric $\left(20^{\circ}\right.$ and $\left.40^{\circ}\right)$ error signals from the hemianopic retina may signify brainstem or residual thalamic processing of peripheral "blind" retinal signals.

Smooth pursuit velocity/target velocity gains were low toward the side of hemispheric ablation in each patient when tracking $20^{\circ}$ triangular waveform targets at frequencies from 0.125 to 1.5 hertz. Mean smooth pursuit gains exceeded unity during tracking to the opposite side at target frequencies up to 1.0 hertz. The high smooth pursuit velocities maintain the target on the seeing hemiretina during tracking toward the blind field. Pursuit was saccadic in both horizontal directions but foveating saccades were directed one way, toward the side of the hemispherectomy. Persisting abnormalities of smooth pursuit and of saccadic latency and trajectory demonstrate limited plasticity of cerebral ocular motor control.

\section{The Clinical-Pathological Relationship of Wernicke's Encephalopathy, \\ Anterograde (LATND) and Retrograde (LRTND) Transneuronal Degeneration in the Limbic System: 8 Case Reports}

William C. Torch, Vancouver

LRTND (sequential retrograde degeneration of anterior thalamus (ATh), mammillothalamic tract (MThT), mammillary body (MB) and fornix $(F x)$ ) following cingulate gyrus lesions was described by Gudden. LATND (anterograde degeneration in Fx, MB, MThT, ATh) following hippocampal ( $\mathrm{Hp}$ ) diseases was recently described and reviewed by Torch et al. TND may occur in Wenicke's encephalopathy (WE). Eight cases of LATND and LRTND in the presence or absence of WE are presented: CASE 1: A 64 y.o. with progressive dementia, memory loss, paranoia and autonomic dysfunction (left $\mathrm{Hp}$ infarction and ipsilateral LATND). $C A S E$ 2: 47 y.o. tuberculous alcoholic with respiratory distress and seizures (left Hp sclerosis and ipsilateral LATND). CASE 3. 51 y.o. tuberculous alcoholic developed mutism and disorientation (right $\mathrm{Hp}$ degeneration with bilateral MB atrophy). $C A S E$ 4: 17 y.o. multiple sclerotic with progressive dementia, sleep disorder and autonomic dysfunction (bilateral $\mathrm{Hp}$ sclerosis and bilateral LATND). CASE 5: 57 y.o. diabetic with progressive OMS (right $\mathrm{MB}$ and anterior commissure infarct with ipsilateral LRTND). CASE 6: 77 y.o. with arteriosclerotic heart disease and progressive dementia (left thalamic and hypothalamic infarct with left LRTND). CASE 7: 61 y.o. cardiac patient ( $\mathrm{Hp}$ infarction and right $\mathrm{MB}$ atrophy diagnosed as unilateral WE). CASE 8: 88 y.o. emphysemic with artersclerosis (multiple infarcts of left $\mathrm{MB}, \mathrm{Fx}$ and $\mathrm{Hp}$ in addition to senile and anoxic Hp changes). The clinical and pathological distinction between LATND, LRTND and WE is sometimes obscure. LATND, LRTND and WE may represent a spectra of time-dependent systems degeneration in Papez's circuit having different etiologies. The degenerative process and symptomatic expression (dementia, hallucinations, psychosis, memory loss and autonomic dysfunction) may be similar. Degeneration may result in direct or indirect loss of hippocampal controls in normal mechanisms of cognition, behaviour and autonomic function.

\section{Recurrent Aseptic Meningitis of Mollaret}

W. J. Becker, S. Coupey, G. Watters and G. A. Ahronheim, Montreal

Three patients with benign recurrent meningitis (of Mollaret) were studied. Onset was at age 27 in one patient, and attacks ceased after 16 years. The two other cases had their first attack in childhood. Both have had multiple episodes of acute aseptic meningitis, and continue to have them. One of these has had a well documented episode of hydrocephalus with increased CSF pressure, papilledema, and bilateral sixth nerve palsies during an attack. All findings returned to normal as the meningitic episode subsided.

Extensive investigations in all three cases have failed to reveal any known etiology for the recurrent attacks.

Although all cases had in common recurrent episodes of headache, nuchal rigidity, and CSF pleocytosis, other features varied widely. The nature of the attacks, their duration, and their interval differed greatly in different patients. One patient consistently showed several thousand polymorphonuclear cells per cubic mm. of spinal fluid early in the attack, with lymphocytes becoming predominant later. Another patient showed only lymphocytes at all stages in her spinal fluid.

The spinal fluid cytology was studied extensively and electron microscopy was done. No evidence of viral particles was seen. Cytological features are discussed, and evidence is presented that the patients who presently fall into the category of Mollaret's meningitis comprise a heterogeneous group with probably diverse etiologies. 